\title{
An integrated vendor-buyer model with quadratic demand under inspection policy and preservation technology
}

\author{
Magfura Pervin ${ }^{1}$ (D), Sankar Kumar Roy ${ }^{* 1}$ (D), Gerhard Wilhelm Weber ${ }^{2,3}$ (D) \\ ${ }^{1}$ Department of Applied Mathematics with Oceanology and Computer Programming, Vidyasagar \\ University, Midnapore-721102, West Bengal, India \\ ${ }^{2}$ Faculty of Engineering Management, Chair of Marketing and Economic Engineering, Poznan University \\ of Technology, ul. Strzelecka 1160-965 Poznan, Poland \\ ${ }^{3}$ IAM (UMA), METU, 06800 Ankara, Turkey
}

\begin{abstract}
An integrated vendor-buyer model for deteriorating items is formulated in this study. To control deterioration rate, the vendor adopts preservation technology. Shortages are allowed for both vendor and buyer. During shortage period, the vendor simply doubles the production rate to meet the demand of buyer. The vendor's demand during non-shortage period follows a constant rate but, the demand is a quadratic decreasing function of time in shortage period. The buyer's demand is a quadratic increasing function of time when shortage does not occur but at shortages period, the demand is constant. The buyer accepts an inspection policy for imperfect product. Total cost is calculated for both the model and integrated system. Thereafter, the model is solved by minimizing the total cost. Numerical examples are given to show the applicability of the model. A sensitivity analysis is done to display the realistic applicability of our model and method.
\end{abstract}

Mathematics Subject Classification (2010). 90B05, 90C26

Keywords. integrated inventory model, quadratic demand, inspection policy, preservation technology, optimization

\section{Introduction}

Most of the companies found that they can obtain further advantages by establishing a long-term relationship between supplier and retailer. Recently, a newly launched mobile sim card is making a huge market from customers in India, where the sim card is delivered by the retailer to the customer, not by the supplier to the customer. But, if there is a misunderstanding between the supplier and retailer, it will be impossible to enjoy such a profit from the customers. So, coordination between partners is a powerful promotional tool to increase the profit and also an efficient key to achieve a global optimality of the system. Hence, an integrated vendor-buyer model is formulated in this paper to obtain

\footnotetext{
*Corresponding Author.

Email addresses: mamoni2014@rediffmail.com (M. Pervin), sankroy2006@gmail.com (S.K. Roy), gerhard.weber@put.poznan.pl (G.W.Weber)

Received: 30.10.2018; Accepted: 27.08.2019
} 
the minimum total relevant cost with a purpose of greater success, rather than by acting separately.

In this highly competitive era, quality of products is an important competition weapon. When a company is manufacturing its own product, the production process is not perfect always. Imperfect production always leads to defective items. When a product is delivered from supplier to retailer, it requires an inspection policy for maintaining the quality and a smooth relationship between supplier and retailer. This imperfect production and inspection policy affects the firms profit negatively during the short- and long- term process. This inspection can cause two types of errors. Type I is the error which occurs if the buyer supplies some imperfect items as perfect. Type II happens if the buyer considers some perfect items as imperfect. Moreover, the imperfect items may be reworked, salvaged or refreshed at a discounted price, which may cause an additional cost. Therefore, imperfectness due to unavoidable factors and two types of inspection policy plays a crucial role in an inventory model.

In many inventory models, it is assumed that the items can be preserved for an infinite time without any change of their physical status. But in reality, many products become partially or totally unusable after a certain time period. Deterioration is defined as (i) pilferage, which is known as physical depletion or evaporation, mainly applicable for petrol, diesel, alcohol or gasoline; (ii) spoilage occurs mainly for vegetables, fish, perishable foodstuffs and fruits; (iii) decay, shown mainly for radioactive substances; (iv) degradation, mainly revealed by pharmaceutical drugs, electronic components, etc. Therefore, the effect of deterioration is vital in our daily life and to be considered in an inventory model.

As described previously, rate of deterioration cannot be removed but the rate of deterioration can be controlled by looking for and developing a preservation technology. For example, the rate of deterioration for fish can be reduced by storing the fish in a deep fridge or by using ice. Applying cool supply-chain policy, the rate of deterioration for fruits becomes less. Though the cost of preservation technology may be high, it will be our attempt to reduce the total cost and to maximize the total profit.

Therefore, every factor described above has a high importance in formulating our model. The main motivation of our work is as follows:

- Deteriorative items are considered; to reduce the rate of deterioration, preservation technology is applied for perishable items.

- Demand for the vendor follows a constant rate during non-shortage period; at the time of shortage, the demand decreases with a progression in time.

- Demand for the buyer during non-shortage period increases with progression in time. At any time interval when shortages occur, the demand decreases as a constant.

- During shortage period, the vendor will double the production to meet the demand of the buyer's and to maintain the reputation of his/her company.

- The buyer takes an inspection policy for checking the items. The non-defective items are separated for selling, and defective items are returned to the vendor at the next shipment. Type I and Type II, both types of errors are inspected at the time of inspection to avoid the penalty cost for delivering imperfect items.

- Shortages are allowed for both vendor and buyer, and only a fraction of backorder is allowed, whereas the rest is lost.

The rest of the paper is organized as follows: Section 2 provides a literature review on previous research about inventory. The notations and assumptions which are used throughout the paper are described in Section 3, which consists of four subsections. In Subsection 3.1, notations that are used for vendor's model are presented. In Subsection 3.2, buyer's notations are stated. In Subsection 3.3, assumptions, which are essential, are listed whereas in Subsection 3.4, buyer's assumptions are given. Section 4 discusses the 
mathematical model of our proposed inventory problem, subdivided in two halves. The vendor's model is displayed in Subsection 4.1, while the buyer's model is derived in Subsection 4.2. Section 5 elaborates the heuristic solution method for the integrated system which consists of four theorems (and instructions) and their proofs. Three illustrative numerical examples are given in Section 6. Section 7 deals with computational experiments, statistical and managerial analysis to identify the important and the further parameters with an exclusive figure. Finally, Section 8 presents the conclusions and further research avenues of our proposed model.

\section{Motivation and review on research}

The integrated vendor-buyer model has received much attention in the last few decades. In particular, Goyal [6] suggested a lot-for-lot policy and that the vendors economic production quantity should be an integer multiple of buyer's purchase quantity. After that, Ouyang et al. [14] addressed a production inventory model with lead time for a singlevendor single-buyer supply chain. Chang et al. [2] discussed an integrated vendor-buyer inventory system under trade-credit policy. An integrated inventory model with transportation for a single-vendor and multi-buyer was described comprehensively by Jha and Shanker [11]. An integrated production-distribution model with probabilistic defect and errors under variable set-up cost was perfectly derived by Priyan and Uthayakumar [22]. Recently, Pervin et al. [20] derived an integrated model with variable holding cost under trade-credit policy. Fauza et al. [5] provided a single-vendor and multiple buyers (SVMB) model to obtain the food inventory policy. They developed a kinetic model, which was utilized to present the quality degradation of the raw material at the vendor.

In a traditional EOQ model, it is assumed that the production process is perfect always and no imperfect items are manufactured. In practice, imperfect items are always rejected by the customers, repaired and reworked by the retailers on the basis of the percentage of imperfection. The repairing process causes an extra charge, which generally means a loss for the supplier. Several researchers considered the effect of imperfectness on production, among them Rosenblatt and Lee [23] who studied an imperfect production process, where the production cycle was optimized. Cárdenas Barrón [1] offered an EPQ model for a single-stage manufacturing system, with a consideration of planned backorders. An EOQ model with imperfect items, shortage and inspection errors, was derived by Hsu and Hsu [9]. Hassan and Diab [7] incorporated a visual inspection policy to test the multiple characteristics simultaneously. Yoo et al. [28] described an inventory model for imperfect items and inspection process with various inspection options under one-time and continuous improvement of investment. Chen [3] formulated an integrated model with production, preventive maintenance, and inspection policy where rework/repair for imperfect items was allowed. A multi-item deteriorating inventory model with trade-credit policy was elaborated by Pervin et al. [21]. Ouyang et al. [13] examined the impacts of collaborative investment and inspection policies on the integrated model of defective items.

Deterioration of products leads to a loss. To derive the sense perfectly, Pervin et al. [17] considered an inventory model for deteriorating items under declining demand market. Deterioration mainly happens due to overstocking or lack of demand. Researchers generally avoid the loss due to deterioration. Avoidance of that loss can causes a huge disaster in the profit of the company. Though cold chain policy can reduce the loss due to deterioration, but it is not always applicable. Recently, Pervin et al. [19] described a model with time-dependent demand and time-varying holding cost including stochastic deterioration. Pervin et al. [18] also studied the effect of deterioration on inventory model with stock-dependent demand under two-level trade credit policy. Recently, Roy et al. [24] proposed a two-warehouse with price discount on backorders and trade-credit policy where the demand function was depicted in probabilistic sense. However, deterioration 
can be controlled by following various Preservation Technology (PT). Rate of deterioration for food, vegetables, fish or fruits can be controlled by applying refrigeration technology. Drying or vacuum technology generally provides a better preserving facility for fruits, flowers, medicine and foodstuff, which usually results in a lower rate of deterioration rate. Ouyang et al. [15] noticed that if the retailer can decrease deteriorating by using the storage facility, the total cost will be lowered. Tsao [25] described that the items with higher deterioration rates produce less profit for the retailer. Yang et al. [26] found out that if the deterioration rate is higher, then more investment is needed for preserving the commodity. Hsu et al. [10] presented PT investment for deteriorating inventory. Dye and Hsieh [4] derived an optimal policy by investing in PT. Yong and Huang [27] found a pricing policy for seasonal products with PT. Mishra et al. [12] presented an inventory model with price- and stock-dependent demand and preservation investment. Hsieh and Dye [8] described a deteriorating model with PT under fluctuating demand. From the above literature studies, one can find a research gap where an integrated model with this type of demand factor, inspection policy and preservation technology for deteriorating items is formulated. To fill the gap, we have derived an integrated inventory model for deteriorating items with inspection policy and preservation technology, where shortages with partial backorder are allowed.

The studies made by research groups related on our topic are surveyed in Table 1.

Table 1: Contributions of some authors related to inventory model.

\begin{tabular}{|c|c|c|c|c|c|c|}
\hline Authors & $\begin{array}{c}\text { Integrated } \\
\text { model }\end{array}$ & $\begin{array}{l}\text { Quadratic } \\
\text { demand }\end{array}$ & $\begin{array}{c}\text { Inspection } \\
\text { policy }\end{array}$ & $\begin{array}{l}\text { Deterio- } \\
\text { rations }\end{array}$ & $\begin{array}{c}\text { Preservation } \\
\text { technology }\end{array}$ & $\begin{array}{c}\text { Partial } \\
\text { backorder }\end{array}$ \\
\hline Ouyang et al. (2015) & $\sqrt{ }$ & & & & & \\
\hline Chang et al. (2009) & $\sqrt{ }$ & & & & & \\
\hline Priyan and Uthayakumar (2017) & $\sqrt{ }$ & & $\sqrt{ }$ & & & \\
\hline Cárdenas-Barrón (2009) & & & $\sqrt{ }$ & & & $\sqrt{ }$ \\
\hline Chen (2013) & & & $\sqrt{ }$ & & $\sqrt{ }$ & \\
\hline Hassan and Diab (2010) & & & $\sqrt{ }$ & & & \\
\hline Hsu and Hsu (2013) & & & $\sqrt{ }$ & & & $\sqrt{ }$ \\
\hline Ouyang et al. (2013) & $\sqrt{ }$ & & $\sqrt{ }$ & & & \\
\hline Yoo et al. (2012) & & & $\sqrt{ }$ & & $\sqrt{ }$ & \\
\hline Dye and Hsieh (2012) & & & & $\sqrt{ }$ & $\sqrt{ }$ & \\
\hline Hsu et al. (2010) & & & & $\sqrt{ }$ & $\sqrt{ }$ & \\
\hline Mishra et al. (2015) & & & & $\sqrt{ }$ & $\sqrt{ }$ & $\sqrt{ }$ \\
\hline Yong and Huang (2013) & & & & $\sqrt{ }$ & $\sqrt{ }$ & \\
\hline Yang et al. (2010) & & & & $\sqrt{ }$ & & $\sqrt{ }$ \\
\hline Pervin et al. (2017) & & & & $\sqrt{ }$ & & $\sqrt{ }$ \\
\hline Pervin et al. (2018) & $\sqrt{ }$ & & & $\sqrt{ }$ & & $\sqrt{ }$ \\
\hline Hsieh and Dye (2013) & & $\sqrt{ }$ & & & $\sqrt{ }$ & \\
\hline This work & $\sqrt{ }$ & $\sqrt{ }$ & $\sqrt{ }$ & $\sqrt{ }$ & $\sqrt{ }$ & $\sqrt{ }$ \\
\hline
\end{tabular}

\section{Notations and assumptions}

For formulating the model, we introduce the following notations and assumptions to execute an understandable form of our work.

\subsection{Vendor's notations}

$\lambda(\alpha) \quad$ deterioration rate per units per time unit when preservation technology is applied;

$\alpha$ unit cost for preservation technology investment per time unit (a decision variable);

$I_{1}(t) \quad$ inventory level for vendor at time $t$ during production period;

$I_{2}(t)$ inventory level for vendor at time $t$ during nonproduction period;

$I_{3}(t) \quad$ inventory level for vendor at time $t$ during shortage period;

$I_{4}(t)$ inventory level for vendor at time $t$ during reproduction period;

$P \quad$ unit production rate per items per unit of time;

$D$ unit demand rate per items per unit of time; 
$Q_{1} \quad$ initial inventory level for vendor;

$m$ number of shipment from vendor to buyer (a decision variable);

$q$ size of each shipment from vendor to buyer (a decision variable);

$A_{1} \quad$ unit ordering cost per items per unit of time;

$c_{1} \quad$ unit fixed cost for transporting items from vendor to buyer;

$c_{2} \quad$ unit variable cost for transporting items from vendor to buyer;

$c_{3}$ unit treatment cost for vendor's per defective item;

$c_{4} \quad$ unit shortage cost per unit;

$d_{1} \quad$ unit deterioration cost per unit;

$h_{1} \quad$ unit holding cost per unit of item per time unit;

$\mu$ proportion of defective items per unit time (a decision variable);

$\delta_{1} \quad$ backorder cost per order;

$V_{T}$ total cost for vendor per unit of time (a decision variable).

\subsection{Buyer's notations}

$I_{5}(t)$ inventory level for buyer at time $t$ during non-shortage period;

$I_{6}(t)$ inventory level for buyer at time $t$ during shortage period;

$Q_{2} \quad$ initial inventory level for buyer;

$q$ size of each shipment received from vendor (a decision variable);

$A_{2} \quad$ unit ordering cost per items per unit of time;

$c_{5} \quad$ unit inspection cost per item per time unit;

$c_{6} \quad$ unit penalty cost for items per unit of time;

$c_{7}$ unit shortage cost;

$d_{2} \quad$ unit deterioration cost per items;

$h_{2}$ unit holding cost for non-defective items per unit per time unit;

$h_{3} \quad$ unit holding cost for defective items per unit per time unit;

$\delta_{2} \quad$ unit lost sale cost;

$B_{T} \quad$ total cost for buyer per unit of time (a decision variable);

$T C$ total cost for the integrated system (a decision variable).

\subsection{Vendor's assumptions}

(1) The vendor applies preservation technology for deteriorating items to reduce the deterioration with a rate $\lambda(\alpha)$, where $\lambda(\alpha)=\lambda_{0} e^{-\delta \alpha}$; here, $\lambda_{0}$ is the deterioration rate without preservation technology and $\delta$ is the parameter of investment for deterioration rate. We assume that the deterioration rate satisfies the equation $\frac{\partial \lambda(\alpha)}{\partial \alpha}<0$ and $\frac{\partial^{2} \lambda(\alpha)}{\partial \alpha^{2}}>0$.

(2) The demand during non-shortage period follows a constant rate $k$, and during shortage period follows a quadratic decreasing function of time. Hence, the demand $D(t)=a_{0}+b_{0} t-c_{0} t^{2}$, where $a_{0}, b_{0}$ and $c_{0}$ are constant.

(3) During shortage period, the vendor will double the production to meet the demand of the buyer's and to maintain the reputation of his company.

(4) Lead time is negligible.

(5) Shortages are allowed, and only a fraction $\beta_{1}, 0 \leq \beta_{1}<1$, is backordered, whereas the rest is lost.

(6) Due to a high demand from buyer, the vendor does not considered inspection policy for their model to save the time and to maintain a good relationship between them by delivering the items at proper time. 


\subsection{Buyer's assumptions}

(1) The demand function for buyer during non-shortage period follows a quadratic increasing function of time and is represented as $D(t)=a+b t+c t^{2}$, where $a, b$ and $c$ are positive constant.

(2) The demand function for the buyer during shortage period follows a constant rate of time, given as $a_{1}$.

(3) As the vendor applies a preservation technology for reducing the deterioration rate, those items when supplied to buyer, the rate of deterioration will be very low, which is taken as $\theta$ where $0<\theta<1$.

(4) The buyer takes an inspection policy for checking the imperfect items to increase the integrated profit and to maintain the overall satisfaction level of the customer. The non-defective items are separated for selling, and the defective items are returned to the vendor at the next shipment.

(5) Shortages are allowed, and only a fraction $\beta_{2}, 0 \leq \beta_{2}<1$, is backordered, whereas the rest is lost.

\section{Mathematical formulation}

In this section, we consider two models, namely, vendor's model which is discussed in Subsection 4.1 and another buyer's model, which is considered in Subsection 4.2.

\subsection{Vendor's model}

At the beginning, the inventory starts with production $P$ and demand $D$. The inventory piles up and reaches to its highest level at time $t=t_{1}$. Then the production stops and the inventory level decreases with the joint effect of demand and deterioration and drops to 0 at time $t=t_{2}$. Shortages are allowed to occur within the interval $\left[t_{2}, t_{3}\right]$ and partially backordered. The demand for vendor during shortage period is considered as a decreasing quadratic function of time. To meet the buyer's demand, the vendor doubles the production rate $P$ during period $\left[t_{3}, t_{4}\right]$. After fulfilling the shortages amount, the inventory level reaches to 0 at time $t=t_{4}$ and then the excesses amount are piles up and the cycle repeats in itself. Now, the instantaneous state of the inventory level is shown by the following differential equations:

$$
\begin{array}{r}
\frac{d I_{1}(t)}{d t}+\lambda(\alpha) I_{1}(t)=P-k \quad\left(t \in\left[0, t_{1}\right]\right) \\
\text { with } I_{1}(0)=0
\end{array}
$$

now, the solution of the differential equation (4.1) by using the boundary condition be

$$
\begin{array}{r}
I_{1}(t)=\frac{P-k}{\lambda(\alpha)}\left(e^{\lambda(\alpha) t}-1\right) \quad\left(t \in\left[0, t_{1}\right]\right) ; \\
\frac{d I_{2}(t)}{d t}+\lambda(\alpha) I_{2}(t)=-k \quad\left(t \in\left[t_{1}, t_{2}\right]\right), \\
\quad \text { with } I_{2}\left(t_{2}\right)=0 ;
\end{array}
$$

solving equation (4.2) with the help of boundary condition, we get

$$
\begin{array}{r}
I_{2}(t)=\frac{k}{\lambda(\alpha)}\left(e^{\lambda(\alpha)\left(t_{2}-t\right)}-1\right) \quad\left(t \in\left[t_{1}, t_{2}\right]\right) ; \\
\frac{d I_{3}(t)}{d t}=-\beta_{1}\left(a_{0}+b_{0} t-c_{0} t^{2}\right) \quad\left(t \in\left[t_{2}, t_{3}\right]\right), \\
\text { with } I_{3}\left(t_{2}\right)=0 ;
\end{array}
$$




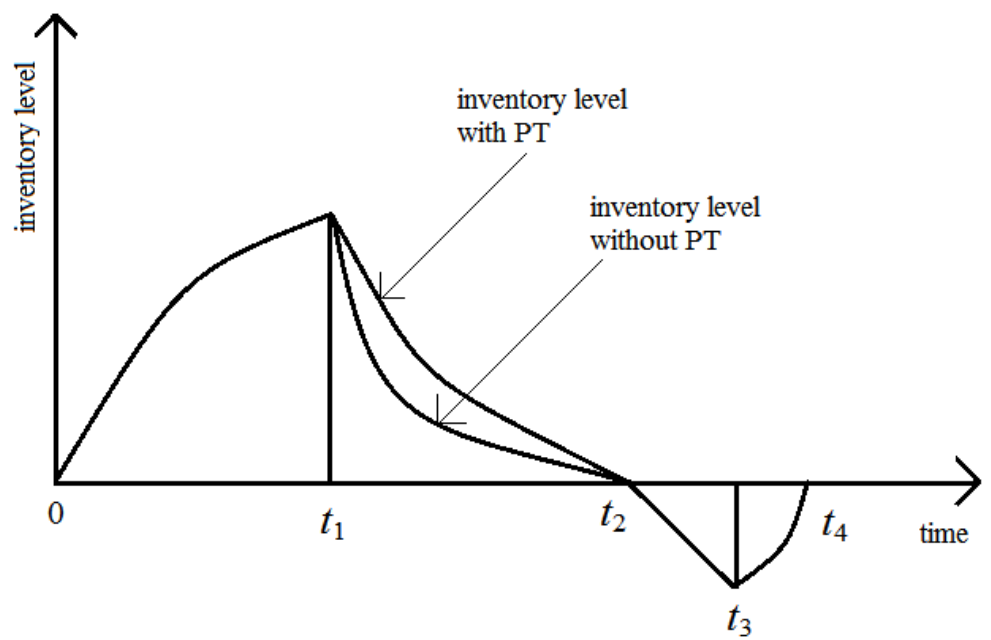

Figure 1. Vendor's inventory model.

solving equation (4.3) with the help of boundary condition, we get

$$
\begin{gathered}
I_{3}(t)=\beta_{1}\left[a_{0}\left(t_{2}-t\right)+\frac{b_{0}}{2}\left(t_{2}^{2}-t^{2}\right)+\frac{c_{0}}{3}\left(t^{3}-t_{2}^{3}\right)\right] \quad\left(t \in\left[t_{2}, t_{3}\right]\right) ; \\
\frac{d I_{4}(t)}{d t}=2 P-\left(a_{0}+b_{0} t-c_{0} t^{2}\right) \quad\left(t \in\left[t_{3}, t_{4}\right]\right) \\
\text { with } I_{3}\left(t_{4}\right)=0
\end{gathered}
$$

solving equation (4.4) with the help of boundary condition, we get

$$
\begin{array}{r}
I_{4}(t)=\left(2 P-a_{0}\right)\left(t-t_{4}\right)+b_{0} \frac{t_{4}^{2}-t^{2}}{2}+c_{0} \frac{t^{3}-t_{4}^{3}}{3}\left(t \in\left[t_{3}, t_{4}\right]\right), \\
Q_{1}=I_{1}\left(t_{1}\right)=\frac{P-D}{\lambda(\alpha)}\left(e^{\lambda(\alpha) t_{1}}-1\right) .
\end{array}
$$

Figure 1 gives a graphical presentation of the vendor's inventory model.

The elements which are important for calculating vendor's total cost are shown below:

(1) The vendor's set-up cost is equal to $m A_{1}$.

(2) The transportation cost for shifting items from vendor to buyer is the sum of a fixed transportation cost per lot and a variable transportation cost. Its value is represented by $m\left(c_{1}+c_{2} m q\right)\left(\int_{0}^{t_{1}} k d t+\int_{t_{1}}^{t_{2}} k d t\right)=m\left(c_{1}+c_{2} m q\right) k t_{2}$.

(3) Total number of pieces of the product that becomes deteriorated during the time interval $\left[0, t_{1}\right]$ and $\left[t_{1}, t_{2}\right]$ is given by

$$
\begin{aligned}
D_{u}=Q_{1}-\int_{0}^{t_{1}} k d t-\int_{t_{1}}^{t_{2}} k d t & =\frac{P-k}{\lambda(\alpha)}\left(e^{\lambda(\alpha) t_{1}}-1\right)-k t_{2} \\
& =\frac{P-k}{\lambda(\alpha)}\left(e^{\lambda(\alpha) t_{1}}-\lambda(\alpha) t_{2}-1\right) .
\end{aligned}
$$

Hence, deterioration cost is represented by $m d_{1} D_{u}=m d_{1} \frac{P-k}{\lambda(\alpha)}\left(e^{\lambda(\alpha) t_{1}}-\lambda(\alpha) t_{2}-1\right)$.

(4) The inventory is available in the system during $\left[0, t_{1}\right]$ and $\left[t_{1}, t_{2}\right]$. Therefore, the holding cost is calculated as

$$
\begin{aligned}
& m h_{1}\left(\frac{q}{P}+\frac{(1-\mu) q}{k}\right)\left(\int_{0}^{t_{1}} I_{1}(t) d t+\int_{t_{1}}^{t_{2}} I_{2}(t) d t\right) \\
& =\frac{m h_{1}}{(\lambda(\alpha))^{2}}\left(\frac{q}{P}+\frac{(1-\mu) q}{D}\right)\left[P\left[e^{\lambda(\alpha) t_{1}}-\lambda(\alpha) t_{1}-1\right]-2 k e^{\lambda(\alpha) t_{1}}+k \lambda(\alpha) t_{2}\right] .
\end{aligned}
$$


(5) In each shipment with size $q, \mu q$ defective items will be returned by the buyer at the end of each shipment cycle. Therefore, the treatment cost for returned defective items per production cycle is of the value $c_{3} m \mu q$.

(6) Preservation technology cost for preserving the deteriorating items is

$$
\begin{aligned}
& \alpha\left(\int_{0}^{t_{1}} I_{1}(t) d t+\int_{t_{1}}^{t_{2}} I_{2}(t) d t\right) \\
& =\frac{\alpha}{(\lambda(\alpha))^{2}}\left[P\left[e^{\lambda(\alpha) t_{1}}-\lambda(\alpha) t_{1}-1\right]-2 k e^{\lambda(\alpha) t_{1}}+k \lambda(\alpha) t_{2}\right] .
\end{aligned}
$$

(7) Shortage cost during the period $\left[t_{2}, t_{3}\right]$ is equal to

$$
\begin{aligned}
& c_{4} \beta_{1} \int_{t_{2}}^{t_{3}}\left(a_{0}+b_{0} t-c_{0} t^{2}\right) d t \\
& =c_{4} \beta_{1}\left[a_{0}\left(t_{3}-t_{2}\right)+b_{0} \frac{\left(t_{3}^{2}-t_{2}^{2}\right)}{2}-c_{0} \frac{\left(t_{3}^{3}-t_{2}^{3}\right)}{3}\right] .
\end{aligned}
$$

(8) Due to shortage during the time interval $\left[t_{2}, t_{3}\right]$, not all of the customers are interested to wait for the coming lot size, which may cause a loss in profit. Hence, lost-sale cost is stated as

$$
\begin{aligned}
\delta_{1}\left(1-\beta_{1}\right) \int_{t_{2}}^{t_{3}} I_{3}(t) d t= & \delta_{1} \beta_{1}\left(1-\beta_{1}\right)\left[\frac{a_{0}}{2}\left(2 t_{2} t_{3}-t_{2}^{2}-t_{3}^{2}\right)+\frac{b_{0}}{6}\left(3 t_{2}^{2} t_{3}-2 t_{2}^{3}-t_{3}^{3}\right)\right. \\
& \left.+\frac{c_{0}}{12}\left(t_{3}^{4}-4 t_{2}^{3} t_{3}+3 t_{2}^{4}\right)\right] .
\end{aligned}
$$

Henceforth, the total cost for the vendor is expressed as

$V_{T}=$ set-up cost + transportation cost + deterioration cost + holding cost + treatment cost + preservation technology cost + shortage cost + lost-sale cost.

Inserting all the parametric values, the total cost for the vendor becomes

$$
\begin{aligned}
V_{T}= & \frac{(1-\mu) q}{k}\left[m A_{1}+m\left(c_{1}+c_{2} m q\right) k t_{1}+m d_{1} \frac{P-k}{\lambda(\alpha)}\left(e^{\lambda(\alpha) t_{1}}-\lambda(\alpha) t_{2}-1\right)\right. \\
& +\frac{m h_{1}}{(\lambda(\alpha))^{2}}\left(\frac{q}{P}+\frac{(1-\mu) q}{D}\right)\left[P\left[e^{\lambda(\alpha) t_{1}}-\lambda(\alpha) t_{1}-1\right]-2 k e^{\lambda(\alpha) t_{1}}+k \lambda(\alpha) t_{2}\right] \\
& +c_{3} m \mu q+\frac{\alpha}{(\lambda(\alpha))^{2}}\left[P\left[e^{\lambda(\alpha) t_{1}}-\lambda(\alpha) t_{1}-1\right]-2 k e^{\lambda(\alpha) t_{1}}+k \lambda(\alpha) t_{2}\right] \\
& +c_{4} \beta_{1}\left[a_{0}\left(t_{3}-t_{2}\right)+b_{0} \frac{\left(t_{3}^{2}-t_{2}^{2}\right)}{2}-c_{0} \frac{\left(t_{3}^{3}-t_{2}^{3}\right)}{3}\right]+\delta_{1} \beta_{1}\left(1-\beta_{1}\right) \\
& {\left[\frac{a_{0}}{2}\left(2 t_{2} t_{3}-t_{2}^{2}-t_{3}^{2}\right)+\frac{b_{0}}{6}\left(3 t_{2}^{2} t_{3}-2 t_{2}^{3}-t_{3}^{3}\right)+\frac{c_{0}}{12}\left(t_{3}^{4}-4 t_{2}^{3} t_{3}+3 t_{2}^{4}\right)\right] . }
\end{aligned}
$$

\subsection{Buyer's model}

After receiving the products from the vendor, at the beginning, the buyer starts to deliver the product as per demand during interval $\left[0, t_{1}\right]$. Shortages occur during interval $\left[t_{1}, t_{2}\right]$ with a constant rate $\beta_{2}$ and a constant demand $a_{1}$. Now, the differential equation of the inventory system is shown as:

$$
\begin{array}{r}
\frac{d I_{5}(t)}{d t}+\theta I_{5}(t)=-\left(a+b t+c t^{2}\right) \quad\left(t \in\left[0, t_{1}\right]\right) \\
\text { with } \quad I_{5}\left(t_{1}\right)=0
\end{array}
$$




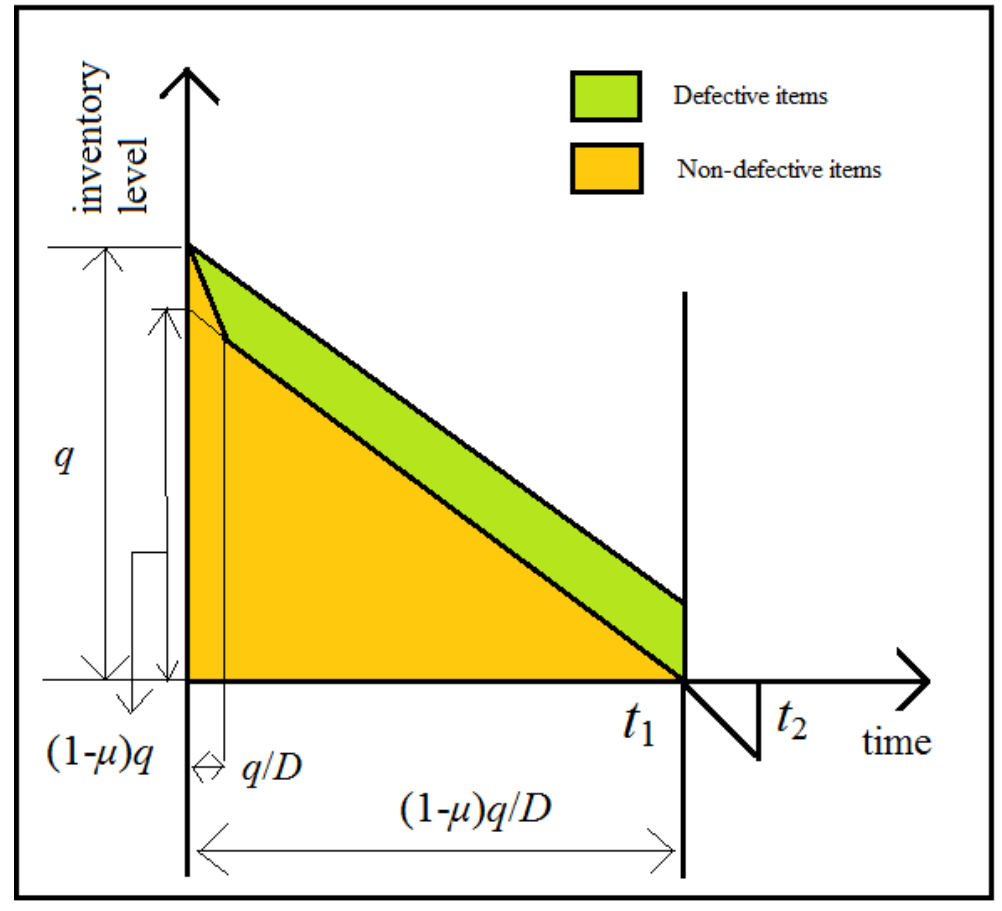

Figure 2. Buyer's inventory model.

now, the solution of the differential equation (4.5) by using the boundary condition be

$$
\begin{aligned}
I_{5}(t)= & \left(\frac{a+b t+c t^{2}}{\theta}-\frac{b+2 c t}{\theta^{2}}+\frac{2 c}{\theta^{3}}\right) e^{\theta\left(t_{1}-t\right)}-\frac{a+b t+c t^{2}}{\theta}+\frac{b+2 c t}{\theta^{2}}-\frac{2 c}{\theta^{3}} ; \\
& \frac{d I_{6}(t)}{d t}=-\beta_{2} a_{1} \quad\left(t \in\left[t_{1}, t_{2}\right]\right), \\
& \text { with } I_{6}\left(t_{1}\right)=0 .
\end{aligned}
$$

solving equation (4.6) with the help of boundary condition, we get

$$
I_{6}(t)=\beta_{2} a_{1}\left(t_{1}-t\right) \quad\left(t \in\left[t_{1}, t_{2}\right]\right) .
$$

Now,

$$
Q_{2}=I_{5}(0)=\left(\frac{a}{\theta}-\frac{b}{\theta^{2}}+\frac{2 c}{\theta^{3}}\right)\left(e^{\theta t_{1}}-1\right)+\left(\frac{b t_{1}+c t_{1}^{2}}{\theta}-\frac{2 c t_{1}}{\theta^{2}}\right) e^{\theta t_{1}} .
$$

The buyer's inventory model is shown in Figure 2.

The elements which are important for calculating buyer's total cost are shown below:

(1) The buyer's ordering cost is of the value $m A_{2}$.

(2) The buyer's inspection cost for checking the imperfect items per lot is $q c_{5} Q_{2}=q c_{5}\left[\left(\frac{a}{\theta}-\frac{b}{\theta^{2}}+\frac{2 c}{\theta^{3}}\right)\left(e^{\theta t_{1}}-1\right)+\left(\frac{b t_{1}+c t_{1}^{2}}{\theta}-\frac{2 c t_{1}}{\theta^{2}}\right) e^{\theta t_{1}}\right]$

(3) Total number of pieces of the product that become deteriorated during the time interval $\left[0, t_{1}\right]$ is given by

$$
\begin{aligned}
D_{u}=Q_{2}-\int_{0}^{t_{1}}\left(a+b t+c t^{2}\right) d t= & \left(\frac{a}{\theta}-\frac{b}{\theta^{2}}+\frac{2 c}{\theta^{3}}\right)\left(e^{\theta t_{1}}-1\right)+ \\
& \left(\frac{b t_{1}+c t_{1}^{2}}{\theta}-\frac{2 c t_{1}}{\theta^{2}}\right) e^{\theta t_{1}}-\left(a t_{1}+\frac{b t_{1}^{2}}{2}+\frac{c t_{1}^{3}}{3}\right) .
\end{aligned}
$$

Therefore, deterioration cost is expressed as

$$
m d_{2} D_{u}=m d_{2}\left[\left(\frac{a}{\theta}-\frac{b}{\theta^{2}}+\frac{2 c}{\theta^{3}}\right)\left(e^{\theta t_{1}}-1\right)+\left(\frac{b t_{1}+c t_{1}^{2}}{\theta}-\frac{2 c t_{1}}{\theta^{2}}\right) e^{\theta t_{1}}-\left(a t_{1}+\frac{b t_{1}^{2}}{2}+\frac{c t_{1}^{3}}{3}\right)\right] .
$$


(4) The inventory is available in the system during $\left[0, t_{1}\right]$. Therefore, the holding cost for non-defective items is calculated as

$$
\begin{aligned}
(1-\mu) q h_{2} \int_{0}^{t_{1}} I_{5}(t) d t= & (1-\mu) q h_{2}\left[\left(\frac{a+b t_{1}+c t_{1}^{2}}{\theta^{2}}-\frac{b+2 c t_{1}}{\theta^{3}}+\frac{2 c}{\theta^{4}}\right)\left(e^{\theta t_{1}}-1\right)\right. \\
& \left.+\left(\frac{b}{\theta^{2}}-\frac{a}{\theta}-\frac{2 c}{\theta^{3}}\right) t_{1}+\left(\frac{c}{\theta^{2}}-\frac{b}{2 \theta}\right) t_{1}^{2}-\frac{c t_{1}^{3}}{3 \theta}\right] .
\end{aligned}
$$

(5) The holding cost for defective items during $\left[0, t_{1}\right]$ is

$$
\begin{aligned}
\mu q h_{3} \int_{0}^{t_{1}} I_{5}(t) d t= & \mu q h_{3}\left[\left(\frac{a+b t_{1}+c t_{1}^{2}}{\theta^{2}}-\frac{b+2 c t_{1}}{\theta^{3}}+\frac{2 c}{\theta^{4}}\right)\left(e^{\theta t_{1}}-1\right)\right. \\
& \left.+\left(\frac{b}{\theta^{2}}-\frac{a}{\theta}-\frac{2 c}{\theta^{3}}\right) t_{1}+\left(\frac{c}{\theta^{2}}-\frac{b}{2 \theta}\right) t_{1}^{2}-\frac{c t_{1}^{3}}{3 \theta}\right] .
\end{aligned}
$$

(6) At the time of inspection, errors can be caused by the buyer. Actually, if the buyer supplies some imperfect items by mistake, he has to pay the penalty cost to the customer. Hence, the penalty cost for items per unit time be

$$
c_{6} \mu q \int_{0}^{t_{1}} D(t) d t=c_{6} \mu q\left[a t_{1}+\frac{b t_{1}^{2}}{2}+\frac{c t_{1}^{3}}{3}\right] .
$$

(7) Shortage cost during the period $\left[t_{1}, T\right]$ is equal to

$$
c_{7} \beta_{2} \int_{t_{1}}^{t_{2}} a_{1} d t=c_{7} \beta_{2} a_{1}\left(t_{2}-t_{1}\right) .
$$

(8) Due to shortage during the time interval $\left[t_{1}, T\right]$, not all of the customers are interested to wait for the coming lot size, which may cause a loss in profit. Hence, lost-sale cost is stated as

$$
\delta_{2}\left(1-\beta_{2}\right) \int_{t_{1}}^{t_{2}} I_{6}(t) d t=\delta_{2}\left(1-\beta_{2}\right) a_{1}\left(t_{2} t_{1}-\frac{t_{2}^{2}+t_{1}^{2}}{2}\right) .
$$

Therefore, the total cost for the buyer is expressed as $B_{T}=$ ordering cost + inspection cost + deterioration cost + holding cost for non-defective items + holding cost for defective items + penalty cost for defective items + shortage cost + lost-sale cost.

Buyer's total relevant cost per unit time is the sum of the described cost, divided by the length of the replenishment cycle $\frac{(1-\mu) q}{D}$; this is expressed by:

$$
\begin{aligned}
B_{T}= & \frac{(1-\mu) q}{D}\left[m A_{2}+q c_{5}\left[\left(\frac{a}{\theta}-\frac{b}{\theta^{2}}+\frac{2 c}{\theta^{3}}\right)\left(e^{\theta t_{1}}-1\right)+\left(\frac{b t_{1}+c t_{1}^{2}}{\theta}-\frac{2 c t_{1}}{\theta^{2}}\right) e^{\theta t_{1}}\right]\right. \\
& +m d_{2}\left[\left(\frac{a}{\theta}-\frac{b}{\theta^{2}}+\frac{2 c}{\theta^{3}}\right)\left(e^{\theta t_{1}}-1\right)+\left(\frac{b t_{1}+c t_{1}^{2}}{\theta}-\frac{2 c t_{1}}{\theta^{2}}\right) e^{\theta t_{1}}-\left(a t_{1}+\frac{b t_{1}^{2}}{2}+\frac{c t_{1}^{3}}{3}\right)\right] \\
& +(1-\mu) q h_{2}\left[\left(\frac{a+b t_{1}+c t_{1}^{2}}{\theta^{2}}-\frac{b+2 c t_{1}}{\theta^{3}}+\frac{2 c}{\theta^{4}}\right)\left(e^{\theta t_{1}}-1\right)+\left(\frac{b}{\theta^{2}}-\frac{a}{\theta}-\frac{2 c}{\theta^{3}}\right) t_{1}\right. \\
& \left.+\left(\frac{c}{\theta^{2}}-\frac{b}{2 \theta}\right) t_{1}^{2}-\frac{c t_{1}^{3}}{3 \theta}\right]+\mu q h_{3}\left[\left(\frac{a+b t_{1}+c t_{1}^{2}}{\theta^{2}}-\frac{b+2 c t_{1}}{\theta^{3}}+\frac{2 c}{\theta^{4}}\right)\left(e^{\theta t_{1}}-1\right)\right. \\
& \left.+\left(\frac{b}{\theta^{2}}-\frac{a}{\theta}-\frac{2 c}{\theta^{3}}\right) t_{1}+\left(\frac{c}{\theta^{2}}-\frac{b}{2 \theta}\right) t_{1}^{2}-\frac{c t_{1}^{3}}{3 \theta}\right]+c_{6} \mu q\left[a t_{1}+\frac{b t_{1}^{2}}{2}+\frac{c t_{1}^{3}}{3}\right] \\
& \left.+c_{7} \beta_{2} a_{1}\left(t_{2}-t_{1}\right)+\delta_{2}\left(1-\beta_{2}\right) a_{1}\left(t_{2} t_{1}-\frac{t_{2}^{2}+t_{1}^{2}}{2}\right)\right] .
\end{aligned}
$$


Now, the total cost for the integrated system becomes

$$
\begin{aligned}
T C= & \frac{(1-\mu) q}{D}\left[m\left(A_{1}+A_{2}\right)+m\left(c_{1}+c_{2} m q\right) k t_{1}+m d_{1} \frac{P-k}{\lambda(\alpha)}\left(e^{\lambda(\alpha) t_{1}}-\lambda(\alpha) t_{2}-1\right)\right. \\
& +\frac{m h_{1}}{(\lambda(\alpha))^{2}}\left(\frac{q}{P}+\frac{(1-\mu) q}{D}\right)\left[P\left[e^{\lambda(\alpha) t_{1}}-\lambda(\alpha) t_{1}-1\right]-2 k e^{\lambda(\alpha) t_{1}}+k \lambda(\alpha) t_{2}\right] \\
& +c_{3} m \mu q+\frac{\alpha}{(\lambda(\alpha))^{2}}\left[P\left[e^{\lambda(\alpha) t_{1}}-\lambda(\alpha) t_{1}-1\right]-2 k e^{\lambda(\alpha) t_{1}}+k \lambda(\alpha) t_{2}\right] \\
& +c_{4} \beta_{1}\left[a_{0}\left(t_{3}-t_{2}\right)+b_{0} \frac{\left(t_{3}^{2}-t_{2}^{2}\right)}{2}-c_{0} \frac{\left(t_{3}^{3}-t_{2}^{3}\right)}{3}\right]+\delta_{1} \beta_{1}\left(1-\beta_{1}\right) \\
& {\left[\frac{a_{0}}{2}\left(2 t_{2} t_{3}-t_{2}^{2}-t_{3}^{2}\right)+\frac{b_{0}}{6}\left(3 t_{2}^{2} t_{3}-2 t_{2}^{3}-t_{3}^{3}\right)+\frac{c_{0}}{12}\left(t_{3}^{4}-4 t_{2}^{3} t_{3}+3 t_{2}^{4}\right)\right] } \\
& +q c_{5}\left[\left(\frac{a}{\theta}-\frac{b}{\theta^{2}}+\frac{2 c}{\theta^{3}}\right)\left(e^{\theta t_{1}}-1\right)+\left(\frac{b t_{1}+c t_{1}^{2}}{\theta}-\frac{2 c t_{1}}{\theta^{2}}\right) e^{\theta t_{1}}\right] \\
& +m d_{2}\left[\left(\frac{a}{\theta}-\frac{b}{\theta^{2}}+\frac{2 c}{\theta^{3}}\right)\left(e^{\theta t_{1}}-1\right)+\left(\frac{b t_{1}+c t_{1}^{2}}{\theta}-\frac{2 c t_{1}}{\theta^{2}}\right) e^{\theta t_{1}}-\left(a t_{1}+\frac{b t_{1}^{2}}{2}+\frac{c t_{1}^{3}}{3}\right)\right] \\
& +(1-\mu) q h_{2}\left[\left(\frac{a+b t_{1}+c t_{1}^{2}}{\theta^{2}}-\frac{b+2 c t_{1}}{\theta^{3}}+\frac{2 c}{\theta^{4}}\right)\left(e^{\theta t_{1}}-1\right)+\left(\frac{b}{\theta^{2}}-\frac{a}{\theta}-\frac{2 c}{\theta^{3}}\right) t_{1}\right. \\
& \left.+\left(\frac{c}{\theta^{2}}-\frac{b}{2 \theta}\right) t_{1}^{2}-\frac{c t_{1}^{3}}{3 \theta}\right]+\mu q h_{3}\left[\left(\frac{a+b t_{1}+c t_{1}^{2}}{\theta^{2}}-\frac{b+2 c t_{1}}{\theta^{3}}+\frac{2 c}{\theta^{4}}\right)\left(e^{\theta t_{1}}-1\right)\right. \\
& \left.+\left(\frac{b}{\theta^{2}}-\frac{a}{\theta}-\frac{2 c}{\theta^{3}}\right) t_{1}+\left(\frac{c}{\theta^{2}}-\frac{b}{2 \theta}\right) t_{1}^{2}-\frac{c t_{1}^{3}}{3 \theta}\right]+c_{6} \mu q\left[a t_{1}+\frac{b t_{1}^{2}}{2}+\frac{c t_{1}^{3}}{3}\right] \\
& \left.+c_{7} \beta_{2} a_{1}\left(t_{2}-t_{1}\right)+\delta_{2}\left(1-\beta_{2}\right) a_{1}\left(t_{2} t_{1}-\frac{t_{2}^{2}+t_{1}^{2}}{2}\right)\right] .
\end{aligned}
$$

\section{Solution procedure}

In this section, we establish some theorems to prove the convexity of the cost function $T C(m, \alpha, \mu, q)$ associated to the integrated system.

Theorem 5.1 (and Instruction). When preservation cost $\alpha$, number of defective items and size of each order are fixed, then the integrated cost function $T C(m, \alpha, \mu, q)$ is convex with respect to ordering frequency $m$.

Proof. The first-order partial derivatives of the integrated cost function $T C(m, \alpha, \mu, q)$ with respect to $m$ is given below:

$$
\begin{aligned}
\frac{\partial T C}{\partial m}= & \left(A_{1}+A_{2}\right)+\left(c_{1}+2 c_{2} m q\right) k t_{1}+d_{1} \frac{P-k}{\lambda(\alpha)}\left[e^{\lambda(\alpha) t_{1}}-\lambda(\alpha) t_{2}-1\right]+\frac{h_{1}}{(\lambda(\alpha))^{2}} \\
& \left(\frac{q}{P}+\frac{(1-\mu) q}{D}\right)\left[P\left[e^{\lambda(\alpha) t_{1}}-\lambda(\alpha) t_{1}-1\right]-2 k e^{\lambda(\alpha) t_{1}}+k \lambda(\alpha) t_{2}\right]+c_{3} \mu q \\
& +d_{2}\left[\left(\frac{a}{\theta}-\frac{b}{\theta^{2}}+\frac{2 c}{\theta^{3}}\right)\left(e^{\theta t_{1}}-1\right)\left(\frac{b t_{1}+c t_{1}^{2}}{\theta}-\frac{2 c t_{1}}{\theta^{2}}\right) e^{\theta t_{1}}-\left(a t_{1}+\frac{b t_{1}^{2}}{2}+\frac{c t_{1}^{3}}{3}\right)\right] .
\end{aligned}
$$


Setting $\frac{\partial T C}{\partial m}=0$ and solving it for optimal $m^{*}$, we obtain:

$$
\begin{aligned}
m^{*}= & \frac{d_{1}(k-P)}{2 c_{2} \lambda(\alpha) q k t_{1}}\left[e^{\lambda(\alpha) t_{1}}-\lambda(\alpha) t_{2}-1\right]-\frac{c_{1}}{2 c_{2} q}-\frac{A_{1}+A_{2}}{2 c_{2} q k t_{1}}+\frac{h_{1}}{2 c_{2}(\lambda(\alpha))^{2} q k t_{1}} \\
& \left(\frac{q}{P}+\frac{(1-\mu) q}{D}\right)\left[P\left[e^{\lambda(\alpha) t_{1}}-\lambda(\alpha) t_{1}-1\right]-2 k e^{\lambda(\alpha) t_{1}}+k \lambda(\alpha) t_{2}\right]-\frac{c_{3} \mu}{2 c_{2} D t_{1}}- \\
& \frac{d_{2}}{2 c_{2} q D t_{1}}\left[\left(\frac{a}{\theta}-\frac{b}{\theta^{2}}+\frac{2 c}{\theta^{3}}\right)\left(e^{\theta t_{1}}-1\right)+\left(\frac{b t_{1}+c t_{1}^{2}}{\theta}-\frac{2 c t_{1}}{\theta^{2}}\right) e^{\theta t_{1}}-\left(a t_{1}+\frac{b t_{1}^{2}}{2}+\frac{c t_{1}^{3}}{3}\right)\right] .
\end{aligned}
$$

The second-order partial derivatives of the integrated cost function $T C(m, \alpha, \mu, q)$ with respect to $m$ is:

$$
\left.\left(\frac{\partial^{2} T C}{\partial m^{2}}\right)\right|_{m=m^{*}}=2 c_{2} q k t_{1}>0 .
$$

Then, we can easily say that $m^{*}$ (optimal $m$, indicating the number of shipment from vendor to buyer) has to be an integer and determination of the optimal $m$ (i.e., $m^{*}$ ) will lead us a local optimal solution. It is noticeable that the cost function $T C(m, \alpha, \mu, q)$ is a convex function of $m$. This completes the proof of the theorem.

Theorem 5.2 (and Instruction). (i) For a known $m$ and fixed $\mu$ and $q, T C(m, \alpha, \mu, q)$ is at its minimum when $\alpha^{*}=0$.

(ii) For that known $m^{*}$, when $\left.\left(\frac{\partial T C}{\partial \alpha}\right)\right|_{m=m^{*}}=0, T C(m, \alpha, \mu, q)$ is convex and has its global minimum at $\alpha^{*}$.

Proof. The first- and second-order partial derivatives of $T C(m, \alpha, \mu, q)$ with respect to $\alpha$ are as follows:

$$
\begin{aligned}
\frac{\partial T C}{\partial \alpha}= & \frac{(1-\mu) q}{D}\left[m d_{1}(P-k) \frac{1}{\lambda(\alpha)}\left(\delta e^{\lambda(\alpha) t_{1}}-\delta \lambda(\alpha) e^{\lambda(\alpha) t_{1}} \lambda_{0}\right)\right]+(P-k) m h_{1} \\
& \left(\frac{q}{P}+\frac{(1-\mu) q}{D}\right)\left[\frac{1}{(\lambda(\alpha))^{2}}\left(2 \delta e^{\lambda(\alpha) t_{1}}-\lambda(\alpha) \delta e^{\lambda(\alpha) t_{1}}\right)-\frac{t_{2}}{(\lambda(\alpha))^{2}}+\frac{2}{(\lambda(\alpha))^{3}}\right] \\
& +\alpha(P-k)\left[\frac{1}{(\lambda(\alpha))^{2}}\left(2 \delta e^{\lambda(\alpha) t_{1}}-\lambda(\alpha) \delta e^{\lambda(\alpha) t_{1}}\right)-\frac{t_{2}}{(\lambda(\alpha))^{2}}+\frac{2}{(\lambda(\alpha))^{3}}\right], \\
\frac{\partial^{2} T C}{\partial \alpha^{2}}= & \frac{(1-\mu) q}{D}\left[m d_{1}(P-k)\left(\frac{1}{\lambda(\alpha)}\left(\delta^{2} e^{\lambda(\alpha) t_{1}}-\delta^{2} \lambda(\alpha) e^{\lambda(\alpha) t_{1}}\right)+\delta^{2} \lambda_{0} \lambda(\alpha) e^{\lambda(\alpha) t_{1}}\right)\right] \\
& +(P-k) m h_{1}\left(\frac{q}{P}+\frac{(1-\mu) q}{D}\right)\left[\frac{1}{(\lambda(\alpha))^{3}}\left(4 \delta^{2} e^{\lambda(\alpha) t_{1}}-2 \delta^{2}(\lambda(\alpha))^{2} e^{\lambda(\alpha) t_{1}}\right)\right. \\
& \left.+\frac{1}{\lambda(\alpha)}\left(\delta^{2} \lambda(\alpha) e^{\lambda(\alpha) t_{1}}-\delta^{2} e^{\lambda(\alpha) t_{1}}\right)+\frac{2 t_{2}}{(\lambda(\alpha))^{3}}-\frac{6}{(\lambda(\alpha))^{4}}\right]+\alpha(P-d) \\
& {\left[\frac{1}{(\lambda(\alpha))^{3}}\left(4 \delta^{2} e^{\lambda(\alpha) t_{1}}-2 \delta^{2}(\lambda(\alpha))^{2} e^{\lambda(\alpha) t_{1}}\right)+\frac{1}{\lambda(\alpha)}\left(\delta^{2} \lambda(\alpha) e^{\lambda(\alpha) t_{1}}-\delta^{2} e^{\lambda(\alpha) t_{1}}\right)\right.} \\
& \left.+\frac{2 t_{2}}{(\lambda(\alpha))^{3}}-\frac{6}{(\lambda(\alpha))^{4}}\right] .
\end{aligned}
$$

Let us define

$$
\begin{aligned}
M(\alpha):= & \frac{(1-\mu) q}{D}\left[m d_{1}(P-k) \frac{1}{\lambda(\alpha)}\left(\delta e^{\lambda(\alpha) t_{1}}-\delta \lambda(\alpha) e^{\lambda(\alpha) t_{1}} \lambda_{0}\right)\right]+(P-k) m h_{1} \\
& \left(\frac{q}{P}+\frac{(1-\mu) q}{D}\right)\left[\frac{1}{(\lambda(\alpha))^{2}}\left(2 \delta e^{\lambda(\alpha) t_{1}}-\lambda(\alpha) \delta e^{\lambda(\alpha) t_{1}}\right)-\frac{t_{2}}{(\lambda(\alpha))^{2}}+\frac{2}{(\lambda(\alpha))^{3}}\right] \\
& +\alpha(P-k)\left[\frac{1}{(\lambda(\alpha))^{2}}\left(2 \delta e^{\lambda(\alpha) t_{1}}-\lambda(\alpha) \delta e^{\lambda(\alpha) t_{1}}\right)-\frac{t_{2}}{(\lambda(\alpha))^{2}}+\frac{2}{(\lambda(\alpha))^{3}}\right]
\end{aligned}
$$


thus,

$$
\begin{aligned}
M^{\prime}(\alpha)= & \frac{(1-\mu) q}{D}\left[\frac{m d_{1}(P-k)}{\lambda(\alpha)}\left(\frac{e^{\lambda(\alpha) t_{1}}}{\lambda(\alpha)}+2 \delta^{2} \lambda(\alpha) e^{\lambda(\alpha) t_{1}} \lambda_{0}-\delta \lambda_{0} e^{\lambda(\alpha) t_{1}}-\delta^{2} e^{\lambda(\alpha) t_{1}} b i g\right)\right] \\
& +\left[(P-k) m h_{1}\left(\frac{q}{P}+\frac{(1-\mu) q}{D}\right)+\alpha(P-k)\right]\left[\frac{2}{(\lambda(\alpha))^{3}}\left(2 e^{\lambda(\alpha) t_{1}}-\lambda(\alpha) e^{\lambda(\alpha) t_{1}}\right)\right. \\
& \left.+\frac{2 \delta^{2} e^{\lambda(\alpha) t_{1}}}{(\lambda(\alpha))^{2}}(\lambda(\alpha)-1)-\frac{2 t_{2}}{\delta(\lambda(\alpha))^{3}}+\frac{6}{\delta(\lambda(\alpha))^{4}}\right]+(P-k)\left[\frac{1}{(\lambda(\alpha))^{2}}\right. \\
& \left.\left(2 \delta e^{\lambda(\alpha) t_{1}}-\lambda(\alpha) \delta e^{\lambda(\alpha) t_{1}}\right)-\frac{t_{2}}{(\lambda(\alpha))^{2}}+\frac{2}{(\lambda(\alpha))^{3}}\right] .
\end{aligned}
$$

Therefore, we study on that

$$
\left.M^{\prime}(\alpha)\right|_{m=m^{*}}>0
$$

Let us introduce,

$$
\begin{aligned}
\Delta_{1}:= & \left.M(\alpha)\right|_{\alpha=0}=\frac{(1-\mu) q}{D}\left[m d_{1}(P-k) \frac{1}{\lambda_{0}}\left(\delta e^{\lambda_{0} t_{1}}-\delta \lambda_{0} e^{\lambda_{0} t_{1}} \lambda_{0}\right)\right]+(P-k) m h_{1} \\
& \left(\frac{q}{P}+\frac{(1-\mu) q}{D}\right)\left[\frac{1}{\lambda_{0}^{2}}\left(2 \delta e^{\lambda_{0} t_{1}}-\lambda_{0} \delta e^{\lambda_{0} t_{1}}\right)-\frac{t_{2}}{\lambda_{0}^{2}}+\frac{2}{\lambda_{0}^{3}}\right]
\end{aligned}
$$

and

$$
\begin{aligned}
\Delta_{2}:= & \left.M(\alpha)\right|_{\alpha=\alpha_{1}}=\frac{(1-\mu) q}{D}\left[m d_{1}(P-k) \frac{1}{\lambda\left(\alpha_{1}\right)}\left(\delta e^{\lambda\left(\alpha_{1}\right) t_{1}}-\delta \lambda\left(\alpha_{1}\right) e^{\lambda\left(\alpha_{1}\right) t_{1}} \lambda_{0}\right)\right] \\
& +(P-k) m h_{1}\left(\frac{q}{P}+\frac{(1-\mu) q}{D}\right)\left[\frac{1}{\left(\lambda\left(\alpha_{1}\right)\right)^{2}}\left(2 \delta e^{\lambda\left(\alpha_{1}\right) t_{1}}-\lambda\left(\alpha_{1}\right) \delta e^{\lambda\left(\alpha_{1}\right) t_{1}}\right)\right. \\
& \left.-\frac{t_{2}}{\left(\lambda\left(\alpha_{1}\right)\right)^{2}}+\frac{2}{\left(\lambda\left(\alpha_{1}\right)\right)^{3}}\right]+\alpha_{1}(P-k)\left[\frac{1}{\left(\lambda\left(\alpha_{1}\right)\right)^{2}}\left(2 \delta e^{\lambda\left(\alpha_{1}\right) t_{1}}-\lambda\left(\alpha_{1}\right) \delta e^{\lambda\left(\alpha_{1}\right) t_{1}}\right)\right. \\
& \left.-\frac{t_{2}}{\left(\lambda\left(\alpha_{1}\right)\right)^{2}}+\frac{2}{\left(\lambda\left(\alpha_{1}\right)\right)^{3}}\right] .
\end{aligned}
$$

Provided, $M^{\prime}(\alpha)>0$, hence, $M(\alpha)$ is strictly increasing in $\alpha$.

Now, it is understandable from the aforementioned observations that:

- If $\Delta_{1} \geq 0$ and $M(\alpha) \geq 0 \forall \alpha \in\left[0, \alpha_{1}\right]$, then $T C(m, \alpha, \mu, q)$ is increasing in $\alpha \in$ $\left[0, \alpha_{1}\right]$. Therefore, the optimal preservation cost is $\alpha^{*}=0$.

- If $\Delta_{1}>0$ and $\Delta_{2}<0$, then by using Intermediate Value Theorem, we can conclude that there exists a unique solution $\alpha \in\left(0, \alpha_{1}\right)$ which satisfies $M\left(\alpha^{*}\right)=0$; this completes the proof.

Theorem 5.3 (and Instruction). There exists a unique $\mu^{*}$ which minimizes the integrated total cost

$T C(m, \alpha, \mu, q)$, when ordering frequency $m$, ordering size $q$ and preservation cost $\alpha$ are fixed. 
Proof. The first-order partial derivative of $T C(m, \alpha, \mu, q)$ with respect to $\mu$ is presented below:

$$
\begin{aligned}
\frac{\partial T C}{\partial \mu}= & \frac{(P-k) m h_{1}}{(\lambda(\alpha))^{2}}\left(\frac{2(\mu-1) q^{2}}{D^{2}}-\frac{q^{2}}{P D}\right)\left[P\left[e^{\lambda(\alpha) t_{1}}-\lambda(\alpha) t_{1}-1\right]-2 k e^{\lambda(\alpha) t_{1}}+k \lambda(\alpha) t_{2}\right] \\
& -\frac{q}{D}\left[m\left(A_{1}+A_{2}\right)+m\left(c_{1}+c_{2} m q\right) k t_{1}+m d_{1} \frac{P-k}{\lambda(\alpha)}\left(e^{\lambda(\alpha) t_{1}}-\lambda(\alpha) t_{2}-1\right)\right] \\
& +\frac{c_{3} m q^{2}(1-2 \mu)}{D}-\frac{q}{D}\left[\frac{\alpha(P-k)}{(\lambda(\alpha))^{2}}\left[P\left[e^{\lambda(\alpha) t_{1}}-\lambda(\alpha) t_{1}-1\right]-2 k e^{\lambda(\alpha) t_{1}}+k \lambda(\alpha) t_{2}\right]\right. \\
& +c_{4} \beta_{1}\left[a_{0}\left(t_{3}-t_{2}\right)+b_{0} \frac{\left(t_{3}^{2}-t_{2}^{2}\right)}{2}-c_{0} \frac{\left(t_{3}^{3}-t_{2}^{3}\right)}{3}\right]+\delta_{1} \beta_{1}\left(1-\beta_{1}\right)\left[\frac { a _ { 0 } } { 2 } \left(2 t_{2} t_{3}-t_{2}^{2}\right.\right. \\
& \left.\left.\left.-t_{3}^{2}\right)+\frac{b_{0}}{6}\left(3 t_{2}^{2} t_{3}-2 t_{2}^{3}-t_{3}^{3}\right)+\frac{c_{0}}{12}\left(t_{3}^{4}-4 t_{2}^{3} t_{3}+3 t_{2}^{4}\right)\right]\right]+q c_{5}\left[\left(\frac{a}{\theta}-\frac{b}{\theta^{2}}\right.\right. \\
& \left.\left.+\frac{2 c}{\theta^{3}}\right)\left(e^{\theta t_{1}}-1\right)+\left(\frac{b t_{1}+c t_{1}^{2}}{\theta}-\frac{2 c t_{1}}{\theta^{2}}\right) e^{\theta t_{1}}\right]+m d_{2}\left[\left(\frac{a}{\theta}-\frac{b}{\theta^{2}}+\frac{2 c}{\theta^{3}}\right)\left(e^{\theta t_{1}}-1\right)\right. \\
& \left.\left.+\left(\frac{b t_{1}+c t_{1}^{2}}{\theta}-\frac{2 c t_{1}}{\theta^{2}}\right) e^{\theta t_{1}}-\left(a t_{1}+\frac{b t_{1}^{2}}{2}+\frac{c t_{1}^{3}}{3}\right)\right]\right]+\frac{2 q^{2} h_{2}(\mu-1)}{D^{2}}\left[\left(\frac{a+b t_{1}+c t_{1}^{2}}{\theta^{2}}\right.\right. \\
& \left.\left.-\frac{b+2 c t_{1}}{\theta^{3}}+\frac{2 c}{\theta^{4}}\right)\left(e^{\theta t_{1}}-1\right)+\left(\frac{b}{\theta^{2}}-\frac{a}{\theta}-\frac{2 c}{\theta^{3}}\right) t_{1}+\left(\frac{c}{\theta^{2}}-\frac{b}{2 \theta}\right) t_{1}^{2}-\frac{c t_{1}^{3}}{3 \theta^{2}}\right]+\frac{q^{2} h_{3}}{D}(1-2 \mu) \\
& {\left[\left(\frac{a+b t_{1}+c t_{1}^{2}}{\theta^{2}}-\frac{b+2 c t_{1}}{\theta^{3}}+\frac{2 c}{\theta^{4}}\right)\left(e^{\theta t_{1}}-1\right)+\left(\frac{b}{\theta^{2}}-\frac{a}{\theta}-\frac{2 c}{\theta^{3}}\right) t_{1}+\left(\frac{c}{\theta^{2}}-\frac{b}{2 \theta}\right) t_{1}^{2}\right.} \\
& \left.-\frac{c t_{1}^{3}}{3 \theta}\right]+\frac{c_{6} q^{2}}{D}(1-2 \mu)\left[a t_{1}+\frac{b t_{1}^{2}}{2}+\frac{c t_{1}^{3}}{3}\right]-\frac{q}{D}\left[c_{7} \beta_{2} a_{1}\left(t_{2}-t_{1}\right)\right. \\
& \left.+\delta_{2}\left(1-\beta_{2}\right) a_{1}\left(t_{2} t_{1}-\frac{t_{2}^{2}+t_{1}^{2}}{2}\right)\right] .
\end{aligned}
$$

Setting $\frac{\partial T C}{\partial \mu}=0$ and solving it for optimal $\mu^{*}$, we get

$$
\begin{aligned}
\mu^{*}= & \frac{D^{2}(\lambda(\alpha))^{2}}{2 q^{2}(P-k) m h_{1}}\left(e^{\lambda(\alpha) t_{1}}-\lambda(\alpha) t_{2}-1\right)^{-1}-\frac{D}{2 c_{3} m q^{2}}+\frac{D^{2}}{2 q^{2} h_{2}}\left[\left(\frac{a+b t_{1}+c t_{1}^{2}}{\theta^{2}}\right.\right. \\
& \left.\left.-\frac{b+2 c t_{1}}{\theta^{3}}+\frac{2 c}{\theta^{4}}\right)\left(e^{\theta t_{1}}-1\right)+\left(\frac{b}{\theta^{2}}-\frac{a}{\theta}-\frac{2 c}{\theta^{3}}\right) t_{1}+\left(\frac{c}{\theta^{2}}-\frac{b}{2 \theta}\right) t_{1}^{2}-\frac{c t_{1}^{3}}{3 \theta}\right] \\
& -\frac{2 q^{2} h_{3}}{D}\left[\left(\frac{a+b t_{1}+c t_{1}^{2}}{\theta^{2}}-\frac{b+2 c t_{1}}{\theta^{3}}+\frac{2 c}{\theta^{4}}\right)\left(e^{\theta t_{1}}-1\right)+\left(\frac{b}{\theta^{2}}-\frac{a}{\theta}-\frac{2 c}{\theta^{3}}\right) t_{1}\right. \\
& \left.+\left(\frac{c}{\theta^{2}}-\frac{b}{2 \theta}\right) t_{1}^{2}-\frac{c t_{1}^{3}}{3 \theta}\right]-\frac{2 c_{6} q^{2}}{D}\left[a t_{1}+\frac{b t_{1}^{2}}{2}+\frac{c t_{1}^{3}}{3}\right]+\frac{D^{2}(\lambda(\alpha))^{2} q c_{5}}{2 q^{2} m h_{1}(P-k)}\left[\left(\frac{a}{\theta}-\frac{b}{\theta^{2}}+\frac{2 c}{\theta^{3}}\right)\right. \\
& \left.\left(e^{\theta t_{1}}-1\right)+\left(\frac{b t_{1}+c t_{1}^{2}}{\theta}-\frac{2 c t_{1}}{\theta^{2}}\right) e^{\theta t_{1}}\right]+m d_{2}\left[\left(\frac{a}{\theta}-\frac{b}{\theta^{2}}+\frac{2 c}{\theta^{3}}\right)\left(e^{\theta t_{1}}-1\right)\right. \\
& \left.\left.+\left(\frac{b t_{1}+c t_{1}^{2}}{\theta}-\frac{2 c t_{1}}{\theta^{2}}\right) e^{\theta t_{1}}-\left(a t_{1}+\frac{b t_{1}^{2}}{2}+\frac{c t_{1}^{3}}{3}\right)\right]\right] .
\end{aligned}
$$


Using the value of $\mu^{*}$ and calculating the second-order partial derivative, we find that

$$
\begin{aligned}
\frac{\partial^{2} T C}{\partial \mu^{2}}= & \frac{2 q^{2}(P-D) m h_{1}}{D^{2}(\lambda(\alpha))^{2}}\left(e^{\lambda(\alpha) t_{1}}-\lambda(\alpha) t_{1}-1\right)-\frac{2 c_{3} m q^{2}}{D}+\frac{2 q^{2} h_{2}}{D^{2}}\left[\left(\frac{a+b t_{1}+c t_{1}^{2}}{\theta^{2}}\right.\right. \\
& \left.\left.-\frac{b+2 c t_{1}}{\theta^{3}}+\frac{2 c}{\theta^{4}}\right)\left(e^{\theta t_{1}}-1\right)+\left(\frac{b}{\theta^{2}}-\frac{a}{\theta}-\frac{2 c}{\theta^{3}}\right) t_{1}+\left(\frac{c}{\theta^{2}}-\frac{b}{2 \theta}\right) t_{1}^{2}-\frac{c t_{1}^{3}}{3 \theta}\right] \\
& -\frac{2 q^{2} h_{3}}{D}\left[\left(\frac{a+b t_{1}+c t_{1}^{2}}{\theta^{2}}-\frac{b+2 c t_{1}}{\theta^{3}}+\frac{2 c}{\theta^{4}}\right)\left(e^{\theta t_{1}}-1\right)+\left(\frac{b}{\theta^{2}}-\frac{a}{\theta}-\frac{2 c}{\theta^{3}}\right) t_{1}\right. \\
& \left.+\left(\frac{c}{\theta^{2}}-\frac{b}{2 \theta}\right) t_{1}^{2}-\frac{c t_{1}^{3}}{3 \theta}\right]-\frac{2 c_{6} q^{2}}{D}\left[a t_{1}+\frac{b t_{1}^{2}}{2}+\frac{c t_{1}^{3}}{3}\right] .
\end{aligned}
$$

Now,

$$
\left(\frac{\partial^{2} T C}{\partial \mu^{2}}\right) \mid\left(\mu=\mu^{*}, \alpha=\alpha^{*}\right)>0 .
$$

Then, we can draw the conclusion that $\mu^{*}$ is the global optimum that minimizes $T C(m, \alpha, \mu, q)$, for fixed values of $\alpha, m$ and $q$. This completes the proof.

Theorem 5.4 (and Instruction). The integrated cost function $T C(m, \alpha, \mu, q)$ is globally convex with respect to ordering size $q$, for fixed $m^{*}, \mu^{*}$ and $\alpha^{*}$.

Proof. The first- and second-order partial derivatives of $T C(m, \alpha, \mu, q)$ with respect to $q$ are given as follows:

$$
\begin{aligned}
& \frac{\partial T C}{\partial q}=\frac{(1-\mu) m}{D}\left(A_{1}+A_{2}\right)+(1-\mu) m t_{1}\left(c_{1}+2 c_{2} m q\right)+\frac{(1-\mu) m d_{1}(P-k)}{D \lambda(\alpha)}\left(e^{\lambda(\alpha) t_{1}}\right. \\
& \left.-\lambda(\alpha) t_{2}-1\right)+\frac{(1-\mu) m h_{1}}{D(\lambda(\alpha))^{2}}\left(\frac{2 q}{P}+\frac{2 q(1-\mu)}{D}\right)\left[P\left[e^{\lambda(\alpha) t_{1}}-\lambda(\alpha) t_{1}-1\right]-2 k e^{\lambda(\alpha) t_{1}}\right. \\
& \left.+k \lambda(\alpha) t_{2}\right]+\frac{2 q(1-\mu) c_{3} m \mu}{D}+\frac{(1-\mu) \alpha(P-k)}{D(\lambda(\alpha))^{2}}\left[P\left[e^{\lambda(\alpha) t_{1}}-\lambda(\alpha) t_{1}-1\right]\right. \\
& \left.-2 k e^{\lambda(\alpha) t_{1}}+k \lambda(\alpha) t_{2}\right]+\frac{(1-\mu) c_{4} \beta_{1}}{D}\left[a_{0}\left(t_{3}-t_{2}\right)+b_{0} \frac{\left(t_{3}^{2}-t_{2}^{2}\right)}{2}-c_{0} \frac{\left(t_{3}^{3}-t_{2}^{3}\right)}{3}\right] \\
& +\frac{(1-\mu)}{D} \delta_{1} \beta_{1}\left(1-\beta_{1}\right)\left[\frac{a_{0}}{2}\left(2 t_{2} t_{3}-t_{2}^{2}-t_{3}^{2}\right)+\frac{b_{0}}{6}\left(3 t_{2}^{2} t_{3}-2 t_{2}^{3}-t_{3}^{3}\right)\right. \\
& \left.+\frac{c_{0}}{12}\left(t_{3}^{4}-4 t_{2}^{3} t_{3}+3 t_{2}^{4}\right)\right]+\frac{2 q(1-\mu) c_{5}}{D}\left[\left(\frac{a}{\theta}-\frac{b}{\theta^{2}}+\frac{2 c}{\theta^{3}}\right)\left(e^{\theta t_{1}}-1\right)\right. \\
& \left.+\left(\frac{b t_{1}+c t_{1}^{2}}{\theta}-\frac{2 c t_{1}}{\theta^{2}}\right) e^{\theta t_{1}}\right]+\frac{(1-\mu) m d_{2}}{D}+\left[\left(\frac{a}{\theta}-\frac{b}{\theta^{2}}+\frac{2 c}{\theta^{3}}\right)\left(e^{\theta t_{1}}-1\right)\right. \\
& \left.+\left(\frac{b t_{1}+c t_{1}^{2}}{\theta}-\frac{2 c t_{1}}{\theta^{2}}\right) e^{\theta t_{1}}-\left(a t_{1}+\frac{b t_{1}^{2}}{2}+\frac{c t_{1}^{3}}{3}\right)\right]+\frac{2 q(1-\mu)^{2} h_{2}}{D}\left[\left(\frac{a+b t_{1}+c t_{1}^{2}}{\theta^{2}}\right.\right. \\
& \left.\left.-\frac{b+2 c t_{1}}{\theta^{3}}+\frac{2 c}{\theta^{4}}\right)\left(e^{\theta t_{1}}-1\right)+\left(\frac{b}{\theta^{2}}-\frac{a}{\theta}-\frac{2 c}{\theta^{3}}\right) t_{1}+\left(\frac{c}{\theta^{2}}-\frac{b}{2 \theta}\right) t_{1}^{2}-\frac{c t_{1}^{3}}{3 \theta}\right] \\
& +\frac{2 q(1-\mu) \mu h_{3}}{D}\left[\left(\frac{a+b t_{1}+c t_{1}^{2}}{\theta^{2}}-\frac{b+2 c t_{1}}{\theta^{3}}+\frac{2 c}{\theta^{4}}\right)\left(e^{\theta t_{1}}-1\right)\right. \\
& \left.+\left(\frac{b}{\theta^{2}}-\frac{a}{\theta}-\frac{2 c}{\theta^{3}}\right) t_{1}+\left(\frac{c}{\theta^{2}}-\frac{b}{2 \theta}\right) t_{1}^{2}-\frac{c t_{1}^{3}}{3 \theta}\right]+\frac{2 q(1-\mu) \mu c_{6}}{D}\left[a t_{1}+\frac{b t_{1}^{2}}{2}+\frac{c t_{1}^{3}}{3}\right] \\
& +\frac{\alpha}{(\lambda(\alpha))^{2}}\left[P\left[e^{\lambda(\alpha) t_{1}}-\lambda(\alpha) t_{1}-1\right]-2 k e^{\lambda(\alpha) t_{1}}+k \lambda(\alpha) t_{2}\right] \\
& +\frac{(1-\mu)}{D}\left[c_{7} \beta_{2} a_{1}\left(t_{2}-t_{1}\right)+\delta_{2}\left(1-\beta_{2}\right) a_{1}\left(t_{2} t_{1}-\frac{t_{2}^{2}+t_{1}^{2}}{2}\right)\right],
\end{aligned}
$$




$$
\begin{aligned}
\frac{\partial^{2} T C}{\partial q^{2}}= & 2 c_{2}(1-\mu) m^{2} t_{1}+\frac{(1-\mu)(P-k) m h_{1}}{D(\lambda(\alpha))^{2}}\left(\frac{2}{P}+\frac{2(1-\mu)}{D}\right)\left(e^{\lambda(\alpha) t_{1}}-\lambda(\alpha) t_{2}-1\right) \\
& +\frac{2(1-\mu) c_{3} m \mu}{D}+\frac{2(1-\mu) c_{5}}{D}\left[\left(\frac{a}{\theta}-\frac{b}{\theta^{2}}+\frac{2 c}{\theta^{3}}\right)\left(e^{\theta t_{1}}-1\right)+\left(\frac{b t_{1}+c t_{1}^{2}}{\theta}-\frac{2 c t_{1}}{\theta^{2}}\right) e^{\theta t_{1}}\right] \\
& +\frac{2(1-\mu)^{2} h_{2}}{D}\left[\left(\frac{a+b t_{1}+c t_{1}^{2}}{\theta^{2}}-\frac{b+2 c t_{1}}{\theta^{3}}+\frac{2 c}{\theta^{4}}\right)\left(e^{\theta t_{1}}-1\right)+\left(\frac{b}{\theta^{2}}-\frac{a}{\theta}-\frac{2 c}{\theta^{3}}\right) t_{1}\right. \\
& \left.+\left(\frac{c}{\theta^{2}}-\frac{b}{2 \theta}\right) t_{1}^{2}-\frac{c t_{1}^{3}}{3 \theta}\right]+\frac{2(1-\mu) \mu h_{3}}{D}\left[\left(\frac{a+b t_{1}+c t_{1}^{2}}{\theta^{2}}-\frac{b+2 c t_{1}}{\theta^{3}}+\frac{2 c}{\theta^{4}}\right)\left(e^{\theta t_{1}}-1\right)\right. \\
& \left.+\left(\frac{b}{\theta^{2}}-\frac{a}{\theta}-\frac{2 c}{\theta^{3}}\right) t_{1}+\left(\frac{c}{\theta^{2}}-\frac{b}{2 \theta}\right) t_{1}^{2}-\frac{c t_{1}^{3}}{3 \theta}\right]+\frac{2(1-\mu) c_{6} \mu}{D}\left[a t_{1}+\frac{b t_{1}^{2}}{2}+\frac{c t_{1}^{3}}{3}\right] .
\end{aligned}
$$

We can see that $\frac{\partial^{2} T C}{\partial q^{2}}>0$ by inserting the values of $m^{*}, \mu^{*}$ and $\alpha^{*}$. Hence, we can easily conclude that $T C(m, \alpha, \mu, q)$ is a strictly convex function of $q$; in fact, there is a global strict convexity. This completes the proof.

Note. We would like to emphasize that Theorems 5.1-5.4 altogether establish and serve as one Multi-Level Procedure. This algorithm has successfully been applied in our applications. To give a higher accuracy to this procedure, both analytically and algorithmically, Implicit Functions (or their approximations) will need to be computed, at anyone of the levels and inserted into the objective function at the following level. However, analytically or numerically, finding those Implicit Functions and working further with them strongly increases the complexity and computational expense of our method. We have studied on this fact, and plan to include this Implicit Functions approach in future applications for special cases, i.e., in less general problem classes.

\section{Numerical examples}

In this section, we solve three problems to give a realistic sense of our proposed model. The numerical data are taken randomly and are validated by using Mathematica with a suitable graph.

Example 6.1. Let us assume: $A_{1}=\$ 100 /$ order, $A_{2}=\$ 200 /$ order, $\lambda_{0}=0.05, T=$ 2 years, $c_{1}=\$ 20 /$ unit, $c_{2}=\$ 15 /$ unit, $c_{3}=\$ 50 /$ unit, $c_{4}=\$ 5 /$ unit $/$ year, $d_{1}=\$ 40 /$ unit, $h_{1}=\$ 50 /$ unit $/$ year, $\delta=0.5, \delta_{1}=\$ 10 /$ order, $c_{5}=\$ 30 /$ unit $/$ year, $c_{6}=\$ 5 /$ unit $/$ year, $c_{7}=$ $\$ 8 /$ unit/year, $d_{2}=\$ 36 /$ unit, $h_{2}=\$ 30 /$ unit/year, $h_{3}=\$ 70 /$ unit/year, $\delta_{2}=\$ 10 /$ order. Then, using Mathematica 9.0, we calculate the values of $m$ for which the integrated cost is minimal. We observe from Table 2 that the integrated cost is at its minimum for $m=4$. Therefore, for $m=4$, we obtain $\alpha^{*}=1.8322, \mu^{*}=21.8719, q=49.1027, V_{T}=6674.25$, $B_{T}=5940.36$ and $T C=4562.91$.

Example 6.2. In this example, we use the same parametric values as above. But, here we consider the case when preservation technology is not allowed. Therefore, the data are: $A_{1}=\$ 100 /$ order, $A_{2}=\$ 200 /$ order, $T=2$ years, $c_{1}=\$ 20 /$ unit, $c_{2}=\$ 15 /$ unit, $c_{3}=$ $\$ 50 /$ unit, $c_{4}=\$ 5 /$ unit/year, $d_{1}=\$ 40 /$ unit, $h_{1}=\$ 50 /$ unit $/$ year, $\delta_{1}=\$ 10 /$ order, $c_{5}=$ $\$ 30 /$ unit/year, $c_{6}=\$ 5 /$ unit/year, $c_{7}=\$ 8 /$ unit/year, $d_{2}=\$ 36 /$ unit, $h_{2}=\$ 30 /$ unit $/$ year, $h_{3}=\$ 70 /$ unit/year, $\delta_{2}=\$ 10 /$ order. Here, we assume: $m=4, \mu^{*}=21.8719$ and $q=$ 49.1027. Then, with the help of Mathematica 9.0, we derive $V_{T}=8526.17, B_{T}=6137.40$ and $T C=5628.54$.

Example 6.3. Now, we recall the same data as in Example 6.1, except that the buyer does not allow an inspection policy on defective items. Then, the required data are: $A_{1}=$ $\$ 100 /$ order, $A_{2}=\$ 200 /$ order, $T=2$ years, $c_{1}=\$ 20 /$ unit, $c_{2}=\$ 15 /$ unit, $c_{3}=\$ 50 /$ unit, $c_{4}=\$ 5 /$ unit $/$ year, $\lambda_{0}=0.05, d_{1}=\$ 40 /$ unit, $h_{1}=\$ 50 /$ unit $/$ year, $\delta_{1}=\$ 10 /$ order, $c_{7}=\$ 8 /$ unit $/$ year, $\delta=0.5, d_{2}=\$ 36 /$ unit, $h_{2}=\$ 30 /$ unit $/$ year, $h_{3}=\$ 70 /$ unit $/$ year, 
$\delta_{2}=\$ 10$ /order. We also employ $m=4, \alpha^{*}=1.8322, \mu^{*}=21.8719$ and $q=49.1027$. By using Mathematica 9.0, we work out the result as $V_{T}=6772.15, B_{T}=7308.49$ and $T C=5822.30$.

Table 2: Computational result.

Bold style represents the optimal solution.

\begin{tabular}{|ccccccc|}
\hline$m$ & $\alpha^{*}$ & $\mu^{*}$ & $q$ & $V_{T}$ & $B_{T}$ & $T C$ \\
\hline 1 & 1.5180 & 20.3428 & 45.6251 & 7225.11 & 6538.60 & 5341.05 \\
\hline 2 & 1.6350 & 20.7926 & 46.5729 & 7618.64 & 6945.68 & 5720.83 \\
\hline 3 & 1.7287 & 21.2476 & 47.6243 & 6927.51 & 6108.47 & 4971.27 \\
\hline $\mathbf{4}$ & $\mathbf{1 . 8 3 2 2}$ & $\mathbf{2 1 . 8 7 1 9}$ & $\mathbf{4 9 . 1 0 2 7}$ & $\mathbf{6 6 7 4 . 2 5}$ & $\mathbf{5 9 4 0 . 3 6}$ & $\mathbf{4 5 6 2 . 9 1}$ \\
\hline 5 & 1.9537 & 22.6773 & 49.6432 & 7180.49 & 6374.12 & 4833.75 \\
\hline 6 & 2.1047 & 22.9844 & 50.5216 & 7319.37 & 6618.81 & 5079.14 \\
\hline 7 & 2.2154 & 23.3560 & 51.2784 & 7538.06 & 6817.25 & 5146.36 \\
\hline 8 & 2.3748 & 23.7836 & 51.7341 & 7726.92 & 7026.57 & 5268.60 \\
\hline 9 & 2.4476 & 24.2608 & 52.3908 & 7941.85 & 7146.72 & 5432.47 \\
\hline 10 & 2.5037 & 24.5738 & 52.8903 & 8047.20 & 7328.08 & 4793.91 \\
\hline 11 & 2.6350 & 24.8902 & 53.2617 & 7841.39 & 7235.55 & 5327.79 \\
\hline 12 & 2.8141 & 25.5437 & 53.5739 & 8140.59 & 7418.93 & 5542.82 \\
\hline
\end{tabular}

\section{Sensitivity analysis}

We now analyze the effects of changes in the system parameters $c_{2}, c_{3}, d_{1}, \delta_{1}, h_{1}, c_{5}$, $c_{6}, d_{2}, h_{2}, h_{3}, \delta_{2}$ on the optimal values of $\alpha^{*}, \mu^{*}$ and the optimal cost $T C$. The sensitivity analysis is performed by changing each of the parameters by $+50 \%,+20 \%,-20 \%$ and $-50 \%$, taking one parameter at a time and keeping the remaining parameters unchanged. The results based on Example 6.1 are shown in Table 3 and, on the basis of these results, the following observations are achieved:

(i) $T C^{*}$ increases moderately with an increase of the vendor's variable transporting cost $c_{2}$ and treatment cost $c_{3}$.

(ii) $T C^{*}$ is more sensitive with regard to change of the values of buyer's inspection cost $c_{5}$ and penalty cost $c_{6}$.

(iii) When the holding cost for both vendor and buyer $\left(h_{1}, h_{2}, h_{3}\right)$ increases, then the optimal cost $T C^{*}$ for the integrated system also increases.

(iv) The value of $T C^{*}$ increases with an increase of the value of vendor's backorder cost $\delta_{1}$ and buyer's lost sale cost $\delta_{2}$.

(v) When the deterioration $\operatorname{costs} d_{1}$ and $d_{2}$ for vendor and buyer decrease, then the integrated cost $T C^{*}$ decreases, respectively.

Based on Table 3, the following managerial insights are settled:

(a) The preservation technology cost is enhanced by increasing the values of $\lambda_{0}$ and $h_{1}$. This implies that when the initial deterioration rate is very high, then the vendor spends more to reduce the deterioration cost. But, when the deterioration rate is very low, then it will be more beneficial if the vendor does not imply a preservation technology. If the holding cost is very high, then the vendor can reduce the cost by ordering more frequently rather than spending on preservation technology.

(b) In all the above cases, if the vendor and buyer invest jointly, then the integrated cost will decrease, which helps to increase the profit of the system.

(c) A higher percentage of defective items in the system will rise the total of the system during each production cycle. At the same time, the number of shipments for repairing damageable items from the buyer to the vendor per lot becomes higher, and also the shipment size increases, which will cause a loss for the buyer. 


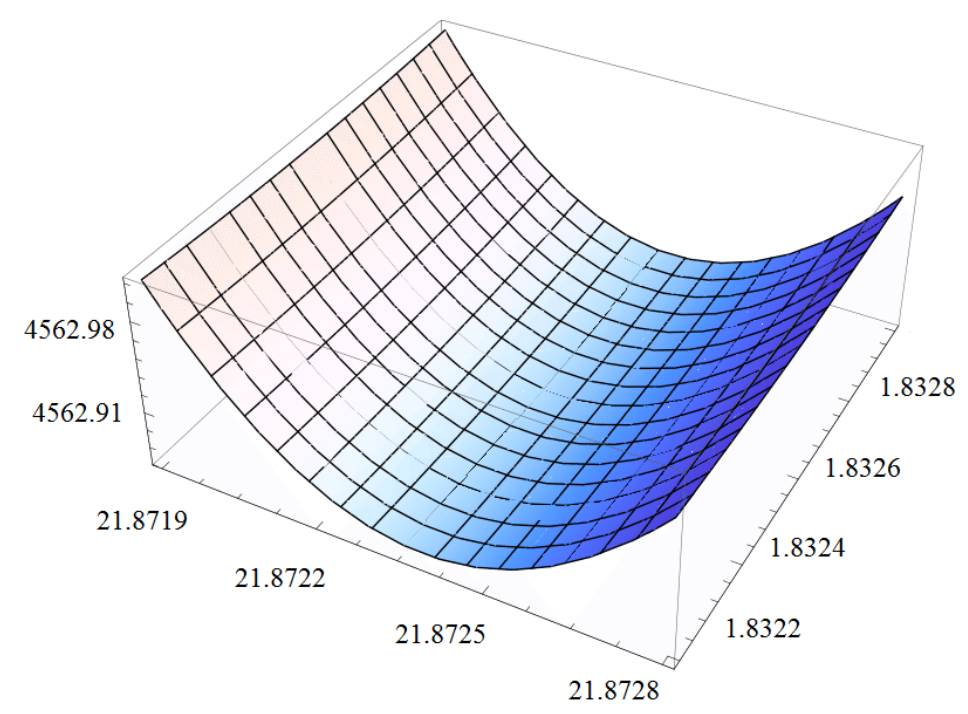

Figure 3. The convexity of the integrated cost function when $m=4$. Included are $\mu^{*}, \alpha^{*}$ and the integrated cost $T C$, along the $x$-axis, the $y$-axis and the $z$-axis, respectively.

Therefore, an inspection policy indirectly helps the buyer to maintain the quality and increase the profit.

(d) It is also found that for low backorder cost, it will be beneficial for the inventory manager to offer the customers a high discount on backorders.

(e) Since preservation technology is applied by the vendor, then the deterioration rate will be lower for the buyer. Therefore, the buyer will order a quite significant amount at a time to reduce the transportation cost.

(f) The model will be applied for perishable food products if the production rate be variable and to be controlled and in that case the assumption that the vendor will doubles the production rate in shortage period should not be applicable. Also, if the products has a short shelf-life, it will be economical for the vendor to extend the expiration date by applying preservation technology through handling, packaging and storing.

Figure 3 shows the required inventory model based on our sensitivity analysis. The function displayed here is of a strictly convex nature. This is fully consistent with our assumptions. Herewith, Figure 3 indicates the feasibility and stability of our offered model. 
Table 3: Sensitivity analysis for different parameter values involved in Example 6.1.

\begin{tabular}{|c|c|c|c|c|c|}
\hline Parameter & $\%$ change & value & $\alpha^{*}$ & $\mu^{*}$ & $T C^{*}$ \\
\hline \multirow{4}{*}{$c_{2}$} & +50 & 22.5 & 2.5479 & 23.3110 & 5714.25 \\
\hline & +20 & 18 & 2.3106 & 22.9257 & 5228.04 \\
\hline & -20 & 12 & 1.8762 & 22.3518 & 4832.91 \\
\hline & -50 & 7.5 & 1.5824 & 21.7997 & 4586.37 \\
\hline \multirow{4}{*}{$c_{3}$} & +50 & 75 & 3.6400 & 25.7508 & 6722.00 \\
\hline & +20 & 60 & 3.2681 & 24.5917 & 6469.74 \\
\hline & -20 & 40 & 2.8930 & 23.7240 & 6153.79 \\
\hline & -50 & 25 & 2.6318 & 22.8637 & 5709.31 \\
\hline \multirow{4}{*}{$d_{1}$} & +50 & 60 & 2.7380 & 23.9463 & 5438.50 \\
\hline & +20 & 48 & 2.5692 & 23.7259 & 5243.16 \\
\hline & -20 & 32 & 2.2435 & 23.4479 & 4973.68 \\
\hline & -50 & 20 & 2.0038 & 23.2108 & 4784.35 \\
\hline \multirow{4}{*}{$\delta_{1}$} & +50 & 15 & 2.4739 & 22.8562 & 5522.24 \\
\hline & +20 & 12 & 2.3392 & 22.7167 & 5317.48 \\
\hline & -20 & 8 & 2.2014 & 22.5874 & 5197.57 \\
\hline & -50 & 5 & 2.1503 & 22.3240 & 4956.33 \\
\hline \multirow{4}{*}{$h_{1}$} & +50 & 75 & 2.9871 & 23.8241 & 5735.72 \\
\hline & +20 & 60 & 2.7432 & 23.6735 & 5523.01 \\
\hline & -20 & 40 & 2.5527 & 23.4106 & 5371.59 \\
\hline & -50 & 25 & 2.3861 & 23.2729 & 5181.46 \\
\hline \multirow{4}{*}{$c_{5}$} & +50 & 45 & 2.7560 & 23.5307 & 5432.88 \\
\hline & +20 & 36 & 2.6178 & 23.4119 & 5394.56 \\
\hline & -20 & 24 & 2.5448 & 23.3627 & 5245.15 \\
\hline & -50 & 15 & 2.4610 & 23.2193 & 5174.38 \\
\hline \multirow{4}{*}{$c_{6}$} & +50 & 7.5 & 1.9142 & 22.095 & 4867.56 \\
\hline & +20 & 6 & 1.8370 & 21.8305 & 4760.64 \\
\hline & -20 & 4 & 1.7552 & 21.5286 & 4692.21 \\
\hline & -50 & 2.5 & 1.6867 & 21.2912 & 4576.49 \\
\hline \multirow{4}{*}{$d_{2}$} & +50 & 54 & 2.1770 & 22.8642 & 5381.06 \\
\hline & +20 & 43.2 & 1.8913 & 22.3473 & 5247.38 \\
\hline & -20 & 28.8 & 21.9867 & 23.3627 & 5160.54 \\
\hline & -50 & 18 & 1.5938 & 21.4654 & 5050.46 \\
\hline \multirow{4}{*}{$h_{2}$} & +50 & 45 & 2.5816 & 23.2100 & 5866.29 \\
\hline & +20 & 36 & 2.4350 & 22.8739 & 5708.16 \\
\hline & -20 & 24 & 2.3864 & 22.1563 & 5581.73 \\
\hline & -50 & 15 & 2.2853 & 21.7469 & 5433.41 \\
\hline \multirow{4}{*}{$h_{3}$} & +50 & 105 & 3.8945 & 24.4610 & 6510.95 \\
\hline & +20 & 84 & 3.7058 & 24.2734 & 6435.64 \\
\hline & -20 & 56 & 3.6467 & 24.0389 & 6372.46 \\
\hline & -50 & 35 & 3.5329 & 23.9113 & 6121.53 \\
\hline \multirow{4}{*}{$\delta_{2}$} & +50 & 15 & 2.5123 & 22.7901 & 5943.00 \\
\hline & +20 & 12 & 2.4042 & 22.6536 & 5810.91 \\
\hline & -20 & 8 & 2.2639 & 22.5407 & 5782.65 \\
\hline & -50 & 5 & 2.1971 & 22.4632 & 5683.39 \\
\hline \multirow{4}{*}{$\delta$} & +50 & 0.75 & 2.8917 & 22.8601 & 6112.46 \\
\hline & +20 & 0.6 & 2.769 & 22.7311 & 5950.79 \\
\hline & -20 & 0.4 & 2.652 & 22.6918 & 5822.37 \\
\hline & -50 & 0.25 & 2.508 & 22.5740 & 5763.28 \\
\hline \multirow{4}{*}{$\lambda_{0}$} & +50 & 0.075 & 3.125 & 25.1423 & 6112.05 \\
\hline & +20 & 0.06 & 2.979 & 24.9050 & 5920.00 \\
\hline & -20 & 0.04 & 2.834 & 24.8247 & 5724.64 \\
\hline & -50 & 0.025 & 2.790 & 24.7339 & 5594.83 \\
\hline
\end{tabular}

\section{Concluding remarks and future study}

In this paper, we have formulated an integrated vendor-buyer model with quadratic demand under inspection policy and preservation technology. The system is considered for both vendor and buyer, and it follows our integrated model. The vendor applies the preservation technology to reduce the loss due to deterioration. However, it is observable from the model that while the initial deterioration rate is very low, spending money on preservation technology will not be beneficial. It is also worth mentioning that if there exists a budget on the investment capital, then there will be a possibility to acquire more 
profit for that organization. The buyer assumes an inspection policy for defective items and the defective items are returned to the vendor for further reparation. The inspection policy is taken by buyer not by vendor because the supplied perfect items can be broken or defective at transportation time. For this reason, the inspection policy taken by buyer will be more effective than vendor and it will also results to increase the integrated profit. Initially, there seems to be a high loss of money; but from our model one can recognize that investing money on inspection policy even improves the production process quality. However, if the buyer's inspection cost is very high, then the buyer does not inspect the received items. Quadratic increasing or decreasing demand is considered for vendor or buyer model because quadratic demand represents both retarded and accelerated growth in demand with respect to time. Also, the most realistic assumptions for cosmetic, seasonal fashion items or high-tech products is appropriately presented by quadratic demand. Two different type of demands are allowed for vendor and buyer model because it covers a vast area of the practical amplified demand function and also helps a company to handle different type of demand faced from real ground. It is also noticeable from our analysis that if vendor and buyer agree on a jointly investment, then the model easily improves the production process.

Our main contributions to literature and to managerial practice are summarized as follows: (i) This paper addresses a preservation technology for deteriorated items and inspection policy for defective items in an integrated model, which was rarely considered in the existing literature. (ii) We develop some useful theorems and prove them for determining the unique optimal solution. (iii) We find that by applying the preservation technology, we can reduce the deterioration rate which increases the total profit for the system. (iv) The model allows for a conclusion that when the buyer follows an inspection policy, it is more profitable to increase the production, while maintaining the quality. (v) Lastly, the model shows that joint investment for vendor and buyer will decrease the cost and increase the profit for the producer.

Regarding further research, we may consider multiple items with multiple replenishment cycles under stochastic demand constraint. For an even more practical situation, one can construct the model by introducing warehouses, quantity discounts, stochastic inflation, deteriorating cost, time-dependent deterioration rate and permissible delay in payments and demand under uncertainty. Consideration of a model with dynamic preservation technology and limited capacity of shelf space will mean another potential extension of our paper. This research can also be continued by inserting unit purchase cost, inventory holding cost and other related factors as time dependent instead of constant. We can also add Collaborative Game Theory by utilizing the model given by Palanci et al. [16] for a more extension of this work. Finally, the model will permit an interesting direction of investigation by incorporating variable or stochastic inflation and time value of money, and with an additional impact by selling defective items at a lower price on demand.

Acknowledgment. The author, Magfura Pervin is very thankful to University Grants Commission (UGC) of India for providing financial support to continue this research work under [MANF(UGC)] scheme: Sanctioned letter number [F1-17.1/2012-13/MANF-201213-MUS-WES-19170 /(SA-III/Website)] dated 28/02/2013.

The research of Gerhard-Wilhelm Weber is partially supported by the Portuguese Foundation for Science and Technology ("FCT-Fundação para a Ciência e a Tecnologia"), through the CIDMA - Center for Research and Development in Mathematics and Applications, within project UID/MAT/ 04106/2013. 


\section{References}

[1] L.E. Cárdenas-Barrón, Economic production quantity with rework process at a singlestage manufacturing system with planned backorders, Comp. Ind. Eng. 57 (3), 11051113, 2009.

[2] H.C. Chang, C.H. Ho, L.Y. Ouyang and C.H. Su, The optimal pricing and ordering policy for an integrated inventory model when trade-credit linked to order quantity, Appl. Math. Model. 33, 2978-2991, 2009.

[3] Y.C. Chen, An optimal production and inspection strategy with preventive maintenance error and rework, J. Manu. Sys. 32 (1), 99-106, 2013.

[4] C.Y. Dye and T.P. Hsieh, An optimal replenishment policy for deteriorating items with effective investment in preservation technology, Euro. J. Oper. Res. 218 (1), 106-112, 2012.

[5] G. Fauza, Y. Amer, S.H. Lee and H. Prasetyo, An integrated single-vendor multibuyer production-inventory policy for food products incorporating quality degradation, Int. J. Prod. Eco. 182, 409-417, 2016.

[6] S.K. Goyal, A joint economic lot size model for purchaser and vendor: A comment, Dec. Sci. 19, 236-241, 1988.

[7] M.H. Hassan and S.L. Diab, Visual inspection of products with geometrical equality characteristics of known tolarences, Ain. Shams. Eng. J. 1 (1), 79-84, 2010.

[8] T.P. Hsieh and C.Y. Dye, A production-inventory model incorporating the effect of preservation technology investment when demand is fluctuating with time, J. Comp. Appl. Math. 239, 25-36, 2013.

[9] J.T. Hsu and L.F. Hsu, An EOQ model with imperfect quality items, inspection errors, shortage backordering, and sales returns, Int. J. Prod. Eco. 143 (1), 162-170, 2013.

[10] P. Hsu, H.M. Wee and H. Teng, Preservation technology investment for deteriorating inventory, Int. J. Prod. Eco. 124 (2), 388-394, 2010.

[11] J.K. Jha and K. Shanker, An integrated-inventory problem with transportation in a divergent supply chain under service-level constraint, J. Manu. Sys. 33, 462-475, 2014.

[12] U. Mishra, L.E. Cárdenas-Barrón, S. Tiwari, A.A. Shaikh and G. Treviño-Garza, An inventory model under price and stock dependent demand for controllable deterioration rate with shortages and preservation technology investment, Annal. Oper. Res. 9, 351-365, 2015.

[13] L.Y. Ouyang, L.Y. Chen and C.T. Yang, Impacts of collaborative investment and inspection policies on the integrated inventory model with defective items, Int. J. Prod. Res. 51 (19), 5789-5802, 2013.

[14] L.Y. Ouyang, C.H. Ho, C.H. Su and C.T. Yang, An integrated inventory model with capacity constraint and ordersize dependent trade-credit, Comp. Ind. Eng. 84, 133$143,2015$.

[15] L.Y. Ouyang, K.S. Wu and C.T. Yang, A study on an inventory model for noninstantaneous deteriorating items with permissible delay in payments, Comp. Ind. Eng. 51 (4), 637-651, 2006.

[16] O. Palanci, S.Z.A. Gok, S. Ergun and G.W. Weber, Cooperative grey games and the grey Shapley value, Optimization. 64 (8), 1-12, 2014.

[17] M. Pervin, G.C. Mahata and S.K. Roy, An inventory model with demand declining market for deteriorating items under trade credit policy, Int. J. Manag. Sci. Eng. Manag. 11 (4), 243-251, 2016.

[18] M. Pervin, S.K. Roy and G.W. Weber, A T wo-echelon inventory model with stockdependent demand and variable holding cost for deteriorating items, Num. Alg. Cont. Opt. 7 (1), 21-50, 2017. 
[19] M. Pervin, S.K. Roy and G.W. Weber, Analysis of inventory control model with shortage under time-dependent demand and time-varying holding cost including stochastic deterioration, Annal. Oper. Res. 260, 437-460, 2018.

[20] M. Pervin, S.K. Roy and G.W. Weber, An integrated inventory model with variable holding cost under two levels of trade-credit policy, Num. Alg. Cont. Opt. 8 (2), 169191, 2018.

[21] M. Pervin, S.K. Roy and G.W. Weber, Multi-item deteriorating two-echelon inventory model with price- and stock-dependent demand: A trade-credit policy., J. Ind. Manag. Opt., 2018, DOI:10.3934/jimo.2018098.

[22] S. Priyan and R. Uthayakumar, An integrated production-distribution inventory system involving probabilistic defective and errors in quality inspection under variable setup cost, Int. Tran. Oper. Res. 24, 1487-1524, 2017.

[23] M.J. Rosenblatt and H.L. Lee, Economic production cycles with imperfect production processes, IIE Tran. 18 (1), 48-55, 1986.

[24] S.K. Roy, M. Pervin and G.W. Weber, A two-warehouse probabilistic model with price discount on backorders under two levels of trade-credit policy, J. Ind. Manag. Opt. 2018, doi:10.3934/jimo.2018167.

[25] Y.C. Tsao, Two-phase pricing and inventory management for deteriorating and fashion goods under trade credit, Math. Meth. Oper. Res. 72 (1), 107-127, 2010.

[26] H.L. Yang, J.T. Teng and M.S. Chern, An inventory model under inflation for deteriorating items with stock-dependent consumption rate and partial backlogging shortages, Int. J. Prod. Eco. 123 (1), 8-19, 2010.

[27] H. Yong and H. Huang, Optimizing inventory and pricing policy for seasonal deteriorating products with preservation technology investment, J. Ind. Eng. 2013, Article ID: 793568, 1-7, 2013.

[28] S.H. Yoo, D. Kim and M.S. Park, Inventory models for imperfect production and inspection processes with various inspection options under one-time and continuous improvement investment, Comp. Oper. Res. 39, 2001-2015, 2012. 